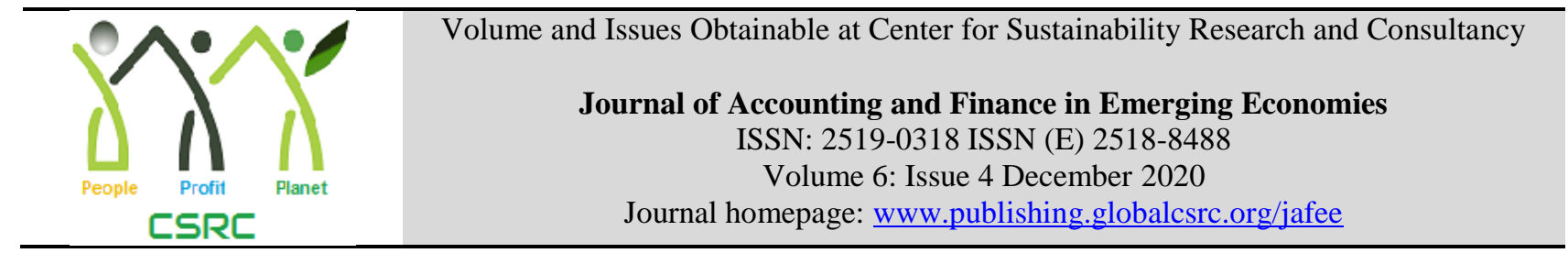

\title{
Corporate Governance and Firm Financial Performance: A Meta-Analysis Study
}

\author{
${ }^{1}$ Waris Ali, ${ }^{2}$ Riaz Hussain Ansari, ${ }^{3}$ Muhammad Abdul Basit Memon \\ ${ }^{1}$ Associate Professor, Department of Business Administration, University of Sahiwal, Sahiwal, Pakistan, \\ waris.ali@uosahiwal.edu.pk \\ ${ }^{2}$ Lecturer, Department of Business Administration, University of Sahiwal, Sahiwal, Pakistan, \\ riazhussain@uosahiwal.edu.pk \\ ${ }^{3}$ Assistant Professor, Department of Business Administration, Sukkur IBA University, Sukkur, Pakistan, \\ basit.memon@iba-suk.edu.pk
}

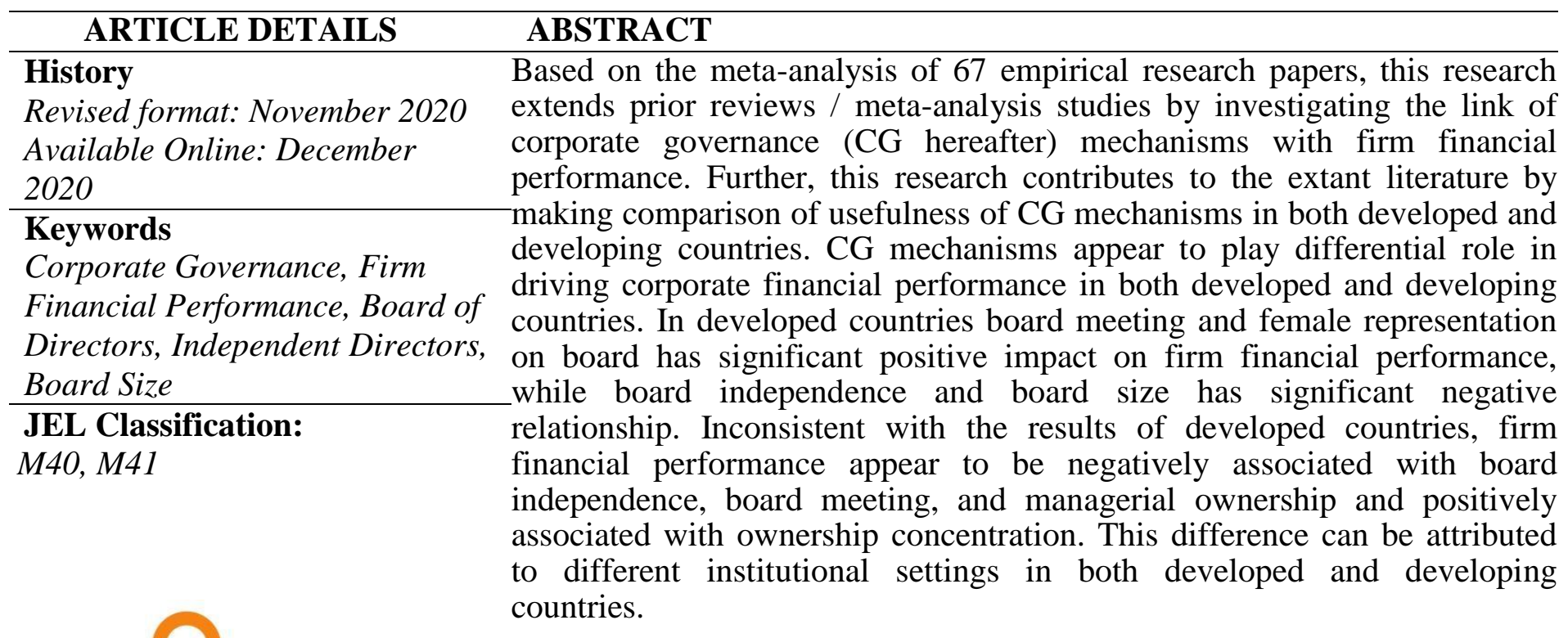

\section{OPEN ACCESS}

(C) 2020 The authors, under a Creative Commons AttributionNonCommercial 4.0

Corresponding author's email address: waris.ali@uosahiwal.edu.pk

Recommended citation: Ali, W., Ansari, R. H. \& Memon, M. A. B. (2020). Corporate Governance and Firm

Financial Performance: A Meta-Analysis Study. Journal of Accounting and Finance in Emerging Economies, 6(4), $917-940$

\section{Introduction}

Corporate scandals notably Enron, Health South Corporation, WorldCom, Tyco, and Lehman Brothers etc. (Agrawal\&Chadha, 2005; Bhagat\& Bolton, 2013 ) and the criminal activities of top level executives in the past three decades triggered the interest of global community in general and the research community in particular regarding CG. Due to the increasing public concern regarding corporate frauds, this concept has now become very popular in academia. The principal motive of CG is to control the opportunistic behavior of top level administrators and executives (Fama\& Jensen, 1983; Sabbaghi, 2016), often found involved in corporate scams. CG is the system to direct and control companies' affairs (Abor, 2007; Alabdullah, 2016). The implementation of good CG mechanisms has led firms to accomplish better financial outcomes (Claessens et al. 2002).

The link between CG mechanisms and corporate financial performance has been empirically investigated by many scholarships (Canyon \& Peck, 1998; Dalton et al., 1999; Guest, 2009; Beiner et al., 2006; Ujunwa, 2012; Palaniappan 
G., 2017). These studies used different proxies such as board committees, board size, board independence, CEO duality, and board meetings (Abor, 2007; Nyamongo \& Temesgen, 2013; Palaniappa G., 2017) to measure CG and used accounting ratios such as return on equity, return on assets and net profit margin and market ratios such as Tobin's Q and earnings per share to measure corporate financial performance (Guest, 2009; Kowalewski, 2012; Darko et al., 2016; Beiner et al., 2006). Further, extant literature was not consistent in using a particular theory to elucidate the linkage between $\mathrm{CG}$ and financial performance and have used different theoretical perspectives such as agency theory, stewardship theory, stakeholder theory, resource dependence theory, and human capital theory (Arora \& Sharma, 2016; Rashid, 2010; Nyamongo \& Temesgen, 2013; Afrifa \& Tauringana, 2015; Vafaei et al., 2015). The existing literature does not provide consensus on the link between CG mechanisms and financial performance for example some studies indicate significant positive relationship between the dimensions of governance and corporate financial performance measures while other show a negative relationship. Therefore, this study aims to draw conclusive results based on the results of existing studies by conducting a meta-analysis on the proxies of CG and firm financial performance in both developed and developing countries. Some meta-analysis studies on the link between CG and firm financial performance have already been conducted but those studies focused on one or two variables to estimate the results (Dalton et al., 1999; 1998) and ignored the other important variables such as such as board size, board independence, board committees, audit committees independence, CEO duality and proportion of female directors on board. Further, corporations in developed and developing countries appear to perceive pressures of different magnitudes from different stakeholders (Ali et al., 2019). It is really precarious to generalize the results of studies conducted in developed countries over developing countries (Gray et al., 1996; De-Villiers and Van-Staden, 2006). This study is therefore targeted to conduct the meta-analysis on the link between CG and firm financial performance by incorporating maximum proxies from studies conducted in both developed and developing countries. This study adds to the extant literature in three ways. Firstly, this study contributes to the literature by incorporating maximum variables of $\mathrm{CG}$ as compared to other meta-analysis studies which focused on one or two variables. Secondly, this study presents literature on CG and corporate financial performance about developed and developing countries in a tabular form which could help the beginners to understand differential impact of CG mechanisms on financial outcomes in developed and developing countries. Finally, this study presents separate meta-analysis results for studies conducted in developed and developing countries.

The remainder paper is structured as follows: The subsequent section consists of literature review, describing studies on the link between CG and corporate financial performance in developed and developing countries. The proceeding section presents research methodology of this research. The penultimate section highlights data analysis results. The last section consists of conclusion, limitations and future research suggestions.

\section{Literature Review}

Previous studies used different classifications to present the existing literature. Dalton et al. (1998) structured existing literature into (1) board size (2) firm's financial performance, and (3) moderating variables. Haniffa and Hudaib (2006) classified their literature into (1) independent/self-reliant variables (2) dependent/reliant variables, and (3) control variables. Shan and McIver (2011) divided their literature into four parts (1) characteristics of CG (2) ownership structures (3) financial indicators, and (4) control variables. In this study we have first divided our literature into developed and developing countries and then further divided into CG and financial measures.

\section{CG and Firm Financial Performance in Developed Countries}

The CG studies conducted in developed countries are summarized in Table 1. Existing studies mainly focused on Australia, US, and UK and paid little attention to other developed countries such as France, Spain, Netherland, Denmark, Italy, Japan, Norway, New Zealand, Germany, and Switzerland (see Table 1). These studies used different theoretical perspectives such as agency theory, stewardship theory, stakeholder theory, and recourse dependence theory but agency theory appeared to be a most dominant theoretical perspective in describing the link between CG and firm financial performance. Further studies extensively relied on secondary data sources and used multiple regression analysis techniques to investigate the relationship between the variables. In the CG category, the most frequently examined determinants of firm financial performance are board size, board independence, board diversity, and shareholder concentration (see Table 2). The studies in developed countries did not provide consensus on the role of board size in deriving corporate financial performance where some studies have shown signification positive 
relationship between board size and corporate financial performance (Beiner et al., 2006; Canyon and Peck, 1998; Rashid and Islam, 2013; Andres et al., 2005;), while some have shown significant negative relationship (Bonn et al., 2004; Guest, 2009). Consistent with board size, other prominent proxies of CG such as board diversity (Bonn et al., 2004; Erhardt et al., 2003), CEO duality (Beiner et al., 2006; Bonn et al., 2004; Christensen et al., 2010; Vafaei et al., 2015), board independence (Bonn et al., 2004; Christensen et al., 2010; Beiner et al., 2006; Vafaei et al., 2015), and shareholder concentration (Christensen et al., 2010; Rashid and Islam, 2013; Gaur et al., 2015; Gedajlovic and Shapiro, 2002) also appear to have differential impact on corporate financial performance. Other CG proxies such as audit committee (Laing and Weir, 1999) and large shareholders (Beiner et al., 2006) also appear to influence corporate financial performance. Corporate financial performance was measured by using different proxies such as return on assets, return on equity, Tobin's Q ratio, earnings per share, net profit margin, and market to book value ratio. However, return on asset and Tobin's Q have appeared to be the most frequently used proxies of corporate financial performance. 


\section{Table 1: CG and Firm Financial Performance in Developed Countries}

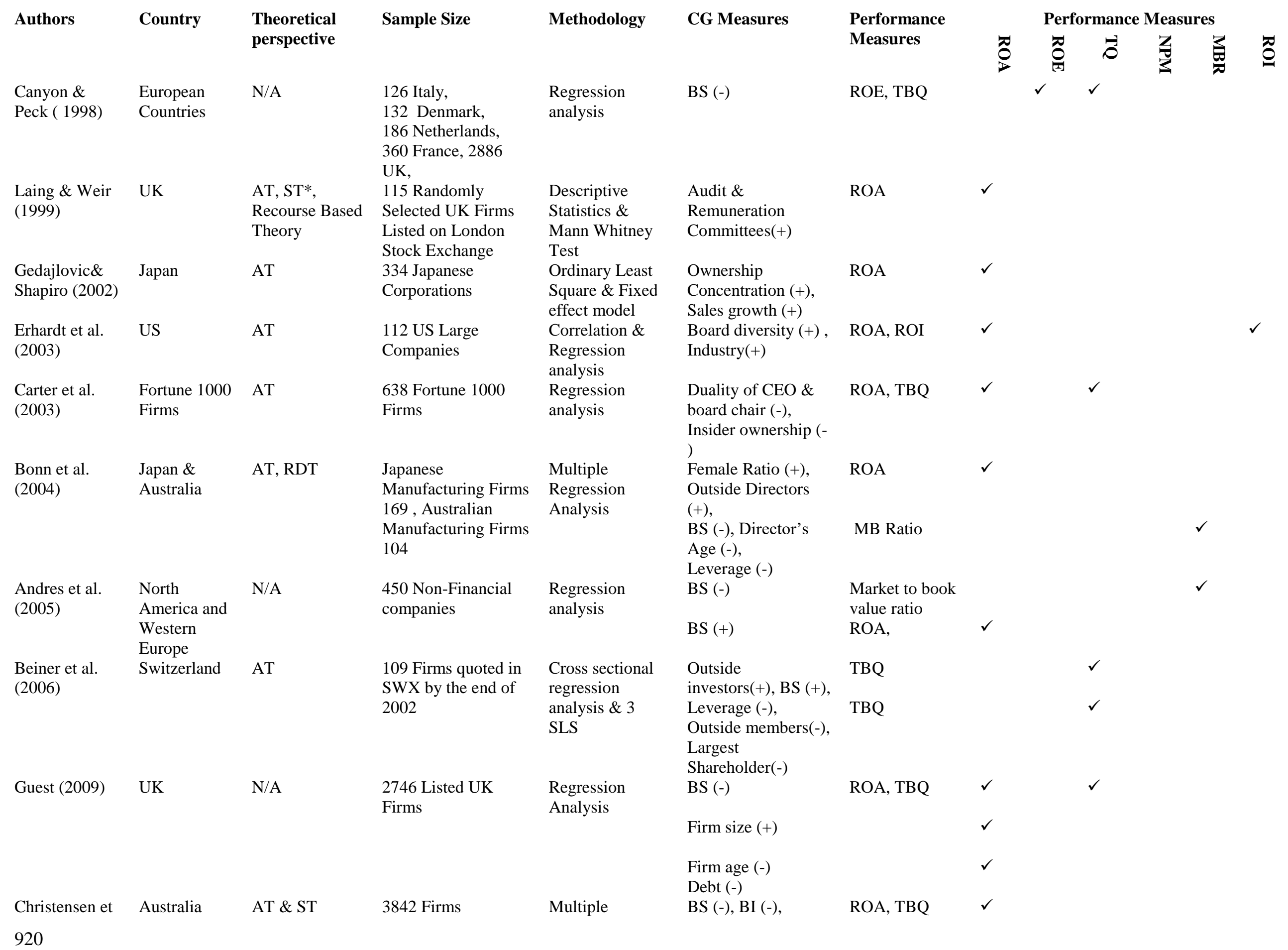


al.

Meca\&Ballest Spain

a (2011)

\section{Rashid \&}

Islam (2013)

Australia

AT

\section{Vafaei et al. Australia} (2015)

Gaur et al. $\begin{array}{ll}\text { New Zealand } & \text { AT, ST, ST*, } \\ & \text { RDT }\end{array}$
$\mathrm{AT} \& \mathrm{ST}^{*}$

6 Firms

(60)

regression

analysis

\section{AT, RDT}

1101 Top 500 ASX listed firms

Analysis

Multiple

regression

analysis

model
Shareholder

Concentration $(+)$,

Firm Size (+),

Leverage (-)

BS (+), BI (-),

Shareholder

Concentration (-),

Firm Size (-),

Leverage $(+)$

Firm Size(-)

Piecewise Linear

Regression

\section{Ownership}

concentration (-),

BS (+), Market

Capitalization (+),

Return on Assets

(+)

OLS regression Board Diversity (+), ROA, ROE,
145 Firms Listed at

New Zealand Stock

Exchange

\section{Random Effect,}

Generalized

Least Square

Estimation \&

Regression

Analysis
BS (-)

Female non-

executive directors

$(+)$, Non-executive

directors (-),

Leverage (-)

Managerial

ownership(+),

Firm Size(-), Blok

holder ownership (-)

Blok holder

ownership (+)

Ownership

Concentration (+),

BS (+), Board

Qualification (+),

CEO duality (-),
TBQ

TBQ, CFO/TA

$$
\text { TBQ, CFO/TA }
$$

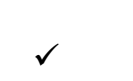

ROA, ROE

Acronyms; AT = Agency Theory; ST = Stakeholder Theory, ST* = Stewardship Theory; RDT = Recourse Dependence Theory; N/A = Not Applied; BS* = Board Structure; BS $=$ Board Size; BM = Board Meetings; BC = Board Committee; NED = Non-Executive Directors; BI = Board Independence; AC = Audit Committee; ACS = Audit Committee Size; $\mathrm{RC}=$ Remuneration Committee $; \mathrm{OC}=$ Ownership Concentration; $\mathrm{NC}=$ Nomination Committee; $\mathrm{IO}=$ Institutional ownership; $\mathrm{SC}=\mathrm{Shareholder}$ Concentration; $\mathrm{MO}=\mathrm{Managerial}$ Ownership, GD = Government Disclosure; IT = Industry Type; FLY = Firm listing year; AT* = Audit Type; ROA = Return on Assets; ROE = Return on Equity; TBQ = Tobin's Q Ratio, EPS = Earnings Per Share, NPM = Net Profit Margin, MBR = Market Book Ratio. 


\section{CG and Financial Performance in Developing Countries}

The studies on the linkage between CG and firm financial performance conducted in developing countries are summarized in table 2. Existing studies mainly focused on Bangladesh, Nigeria, Thailand, Malaysia, Ghana, China, India, Pakistan, Hong Kong, UAE, Saudi Arabia, and Srilanka (see Table 2). Consistent with the findings of developed countries, studies in developing countries also used different theories to explain the link between CG and financial performance and predominantly relied on agency theoretical perspective. Likewise in developed countries, studies extensively relied on secondary data sources and used multiple regression analysis techniques to investigate the relationship between variables. Consistent with the developed countries board size, board independence, and CEO duality appeared to be the most frequently used proxies of CG (see Table 2). These studies does not provide consensus on the role of board size, board independence, and CEO duality in deriving corporate financial performance and appear to have differential impact (see Yammeesri and Herath, 2010; Rashid et al., 2010; Rashid, 2010; Zulkafli and Samad, 2007; Lei and Song, 2012; Lam and Lee, 2012; Sheikh et al., 2013; Haniffa and Hudaib, 2006). Contrary to the findings of studies in developed countries, studies in developing countries used relatively a large number of proxies such as managerial ownership, institutional ownership, government ownership, foreign ownership, concentrated ownership, board composition, female representation on board, and multiple directorships to measure the CG. These proxies appear to show different kind of impact on financial performance. In addition to the above, firm size, age, leverage, and industry also appear to influence corporate financial performance. Consistent with the results in developed countries, corporate financial performance was measured by using different proxies such as return on equity, return on assets, Tobin's Q ratio, earnings per share, net profit margin, and market to book value ratio; and return on asset and Tobin's Q appeared to be the most frequently used proxies of corporate financial performance. 
Table 2: CG and Firm Financial Performance in Developing Countries

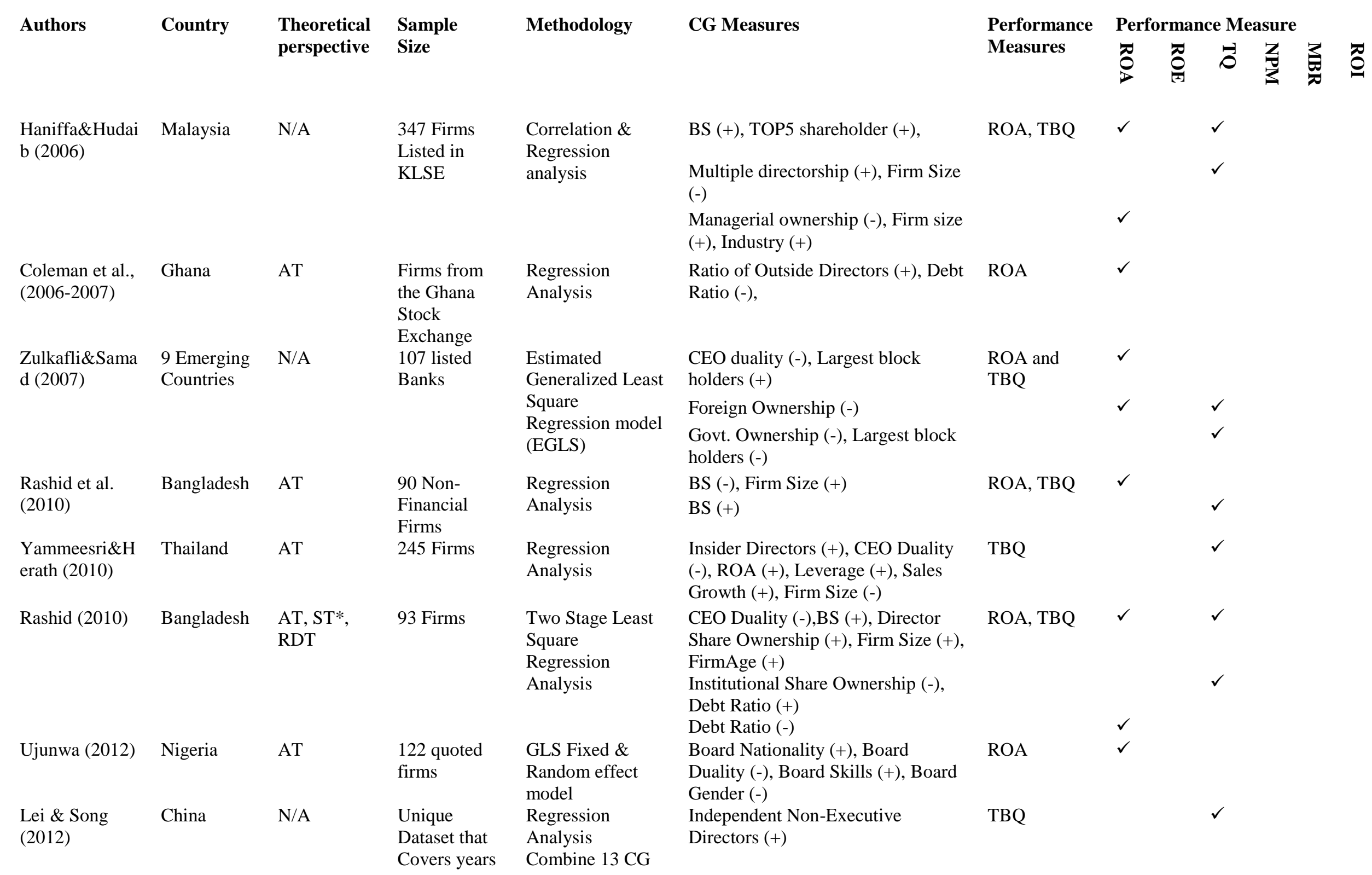




\begin{tabular}{|c|c|c|c|c|c|c|c|c|c|}
\hline Authors & Country & Theoretical & Sample & & CG Measures & Performance & & orma & ce Me \\
\hline & & & $\begin{array}{l}\text { From } 2001 \text { to } \\
2009\end{array}$ & $\begin{array}{l}\text { Attributes Using } \\
\text { PCA }\end{array}$ & & & & & \\
\hline $\begin{array}{l}\text { Lam \& Lee } \\
(2012)\end{array}$ & Hong Kong & AT & $\begin{array}{l}346 \text { Firm } \\
\text { Year } \\
\text { Observations } \\
\text { of public } \\
\text { firms }\end{array}$ & $\begin{array}{l}\text { Multiple } \\
\text { Regression } \\
\text { Analysis }\end{array}$ & $\begin{array}{l}\text { Remuneration Committee in family } \\
\text { firms (-), Nomination Committee } \\
(+), \text { CEO duality }(+)\end{array}$ & ROA, ROE & $\checkmark$ & $\checkmark$ & \\
\hline $\begin{array}{l}\text { Mollah et } \\
\text { al.(2012) }\end{array}$ & $\begin{array}{l}\text { Asian } \\
\text { Emerging } \\
\text { Markets }\end{array}$ & AT & $\begin{array}{l}\text { 19Companies } \\
\text { Listed in } \\
\text { Botswana } \\
\text { Stock Market } \\
(2000-2007)\end{array}$ & $\begin{array}{l}\text { Ordinary Least } \\
\text { Square Models }\end{array}$ & Foreign ownership (+), Industry (+) & $\begin{array}{l}\text { ROA, ROE, } \\
\text { TBQ }\end{array}$ & $\checkmark$ & $\checkmark$ & $\checkmark$ \\
\hline $\begin{array}{l}\text { Sheikh et } \\
\text { al.(2013) }\end{array}$ & Pakistan & $\begin{array}{l}\text { AT, ST, } \\
\text { RDT }\end{array}$ & 154 Firms & $\begin{array}{l}\text { Panel econometric } \\
\& \text { polled ordinary } \\
\text { least square } \\
\text { method }\end{array}$ & $\begin{array}{l}\text { BS (+), Non-Executive Directors (- } \\
\text { ), Managerial Ownership (-), } \\
\text { Ownership Concentration (+), Firm } \\
\text { Size (+), Leverage (-) }\end{array}$ & $\begin{array}{l}\text { ROA, ROE, } \\
\text { EPS, MBR. }\end{array}$ & $\checkmark$ & $\checkmark$ & \\
\hline $\begin{array}{l}\text { Hassan } \\
\& \text { Halbouni } \\
(2013)\end{array}$ & UAE & AT & 95 Firms & $\begin{array}{l}\text { Regression } \\
\text { analysis }\end{array}$ & BS (+), CEO Duality (+) & $\begin{array}{l}\text { ROA, ROE, } \\
\text { Tobin Q }\end{array}$ & $\checkmark$ & $\checkmark$ & \\
\hline $\begin{array}{l}\text { Manawaduge\& } \\
\text { Zoysa (2013) }\end{array}$ & Sri Lanka & AT & 157 Firms & $\begin{array}{l}\text { Regression } \\
\text { analysis }\end{array}$ & $\begin{array}{l}\text { Ownership Concentration (-) } \\
\text { Ownership Structure (+) }\end{array}$ & $\begin{array}{l}\text { ROA, ROE, } \\
\text { TBQ, MBR }\end{array}$ & $\begin{array}{l}\checkmark \\
\checkmark\end{array}$ & $\checkmark$ & \\
\hline $\begin{array}{l}\text { Nyamongo\&Te } \\
\text { mesgen }(2013)\end{array}$ & Kenya & AT \& ST* & $\begin{array}{l}37 \\
\text { Commercial } \\
\text { Banks }\end{array}$ & $\begin{array}{l}\text { Panel } \\
\text { Econometric } \\
\text { model }\end{array}$ & BS (-), Independent directors (+) & ROA, ROE & $\checkmark$ & $\checkmark$ & \\
\hline Li et al. (2015) & China & N/A & $\begin{array}{l}1241 \text { firms } \\
\text { listed on } \\
\text { Shanghai } \\
\text { stock } \\
\text { exchange }\end{array}$ & $\begin{array}{l}\text { Regression } \\
\text { analysis }\end{array}$ & $\begin{array}{l}\text { Independent directors (-), } \\
\text { Ownership Concentration (-) }\end{array}$ & ROA, TBQ & $\checkmark$ & & $\checkmark$ \\
\hline $\begin{array}{l}\text { Pamburai et al. } \\
\text { (2015) }\end{array}$ & $\begin{array}{l}\text { South } \\
\text { Africa }\end{array}$ & N/A & 158 Firms & $\begin{array}{l}\text { Multiple } \\
\text { regression } \\
\text { analysis }\end{array}$ & $\begin{array}{l}\text { Non-executive directors (+) } \\
\text { Board Meeting (-), } \\
\text { Firm Size (+), Leverage (-) }\end{array}$ & $\begin{array}{l}\text { EVA, ROA, } \\
\text { TBQ }\end{array}$ & $\begin{array}{l}\checkmark \\
\checkmark\end{array}$ & & $\begin{array}{l}\checkmark \\
\checkmark\end{array}$ \\
\hline $\begin{array}{l}\text { Basyith et al., } \\
\text { (2015) }\end{array}$ & Indonesia & AT & $\begin{array}{l}38 \text { Firms } \\
\text { Listed in } \\
\text { Indonesian } \\
\text { Stock } \\
\text { Exchange }\end{array}$ & $\begin{array}{l}\text { Tobit Regression } \\
\text { Analysis }\end{array}$ & $\begin{array}{l}\text { Managerial Ownership (-), Block } \\
\text { holder Ownership (+), BS (-) }\end{array}$ & ROA & $\checkmark$ & & \\
\hline
\end{tabular}




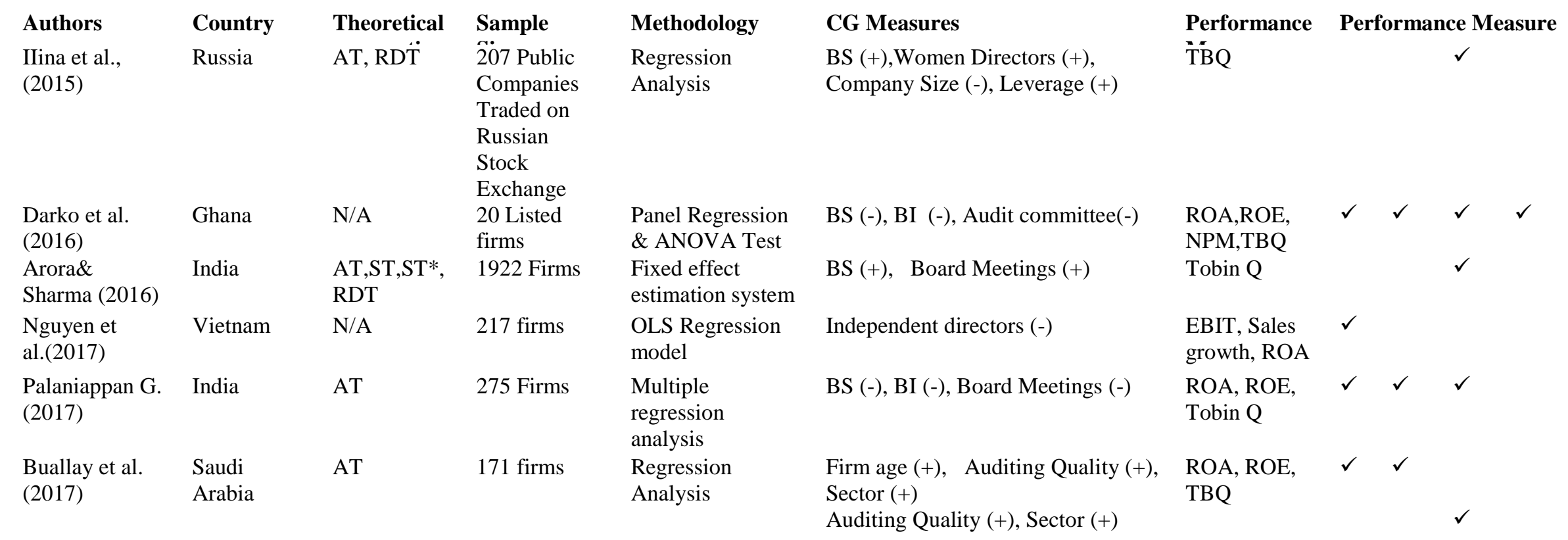

AT $=$ Agency Theory; ST $=$ Stakeholder Theory; ST $*=$ Stewardship Theory; RDT $=$ Resource Dependence Theory; N $/$ A $=$ Not Applied; BS $=$ Board Size; BC $=$ Board Committee; BS* = Board Structure; BM = Board Meetings; NED = Non-Executive Directors; BI = Board Independence; ACS = Audit Committee Size; RC = Remuneration Committee; $\mathrm{AC}=$ Audit Committee; $\mathrm{NC}=$ Nomination Committee; $\mathrm{IO}=$ Institutional Ownership; OC = Ownership Concentration; MO = Managerial Ownership; SC = Shareholder Concentration; GD = Government Disclosure; IT = Industry Type; FLY = Firm listing year; AT* = Audit type; ROA = Return on Assets; ROE = Return on Equity; TBQ = Tobin's Q Ratio; EPS = Earnings Per Share; NPM = Net Profit Margin; MBR = Market Book Ratio. 


\section{Methodology}

Meta-analysis is a widely accepted approach to quantitatively integrate the results from the extant literature (Dalton et al., 1999; Combs et al., 2011). We have conducted this Meta-analysis by following the guidelines provided by Ali et al. (2015). Meta-analysis enables researchers to determine the true association between two variables (Dalton et al., 1999). To accomplish this purpose, we collected information about sample size and correlation coefficient to calculate the weighted correlation coefficient and corrected correlation coefficient between the variables.

At first, we have used different keywords such as 'corporate governance structure and firm financial performance', 'board characteristics and firm financial performance', 'board size and firm financial performance', 'CEO duality and firm financial performance', 'board independence and firm financial performance', 'board committees and firm financial performance', 'ownership structure and firm financial performance' to search relevant articles on the topic under study. We have searched these keywords by using different online databases such as Google Scholar, Emerald, Science Direct, and Jstor. We have also examined the reference list of available articles and find more articles on the topic under study. At the second stage, we have filtered available studies according to the information required. In order to improve the quality of Meta-analysis we have only shortlisted studies of those journals enlisted in ABS ranking. In the third stage, we ensured that shortlisted studies, examining the link between CG and firm financial performance, are empirical and quantitative in nature. As a result we come up with 67 empirical studies.

At the fourth stage, we used correlation coefficient, a measure of relationship between CG and firm performance, as a unit of analysis. Therefore we searched correlation coefficient from all the selected studies. After searching for the key terms, we have prepared a comprehensive table summarizing studies into developed (see Table 1) and developing (see Table 2) countries. After that we calculated weighted correlation coefficient. To calculate weighted correlation coefficient we, firstly, sum up all the ratios and divided them by number of ratios to take average correlation (R). Then we multiply the average correlation with the sample size of corresponding study which results in weighted correlation coefficient $(\mathrm{Rn})$. This weighted correlation coefficient is used to estimate the overall average corrected correlation coefficient (Rc).We used the following formula for the calculation of average corrected correlation coefficient (Ali et al. 2015).

$\mathrm{Rc}=\sum \mathrm{R}_{\mathrm{i}} \mathrm{n}_{\mathrm{i}} / \mathrm{N}$

Where, $\mathrm{R}_{\mathrm{i}}$ is the correlation coefficient of individual firms, $\mathrm{n}_{\mathrm{i}}$ is the firms' corresponding sample size and $\mathrm{N}$ is overall sample size of all studies. The $\mathrm{p}$ value for each $\mathrm{Rc}$ is calculated from the internet by putting values of $\mathrm{R}$ score and overall sample size $(\mathrm{N})$.

\section{Data Analysis}

This section describes the Meta analysis of variables for both developed and developing countries.

\subsection{Meta-Analysis for the studies in developed countries}

In developed countries, the variables for which the data was available were included into the analysis. Those variables are board independence, board size, board meetings, female representation on board, firm size, firm age, leverage and industry. Table 3 to Table 10 presents one by one meta-analysis of those variables. Meta-analysis result shows that board meetings and female representation on board have significant positive relationship with firm financial performance, while board size, firm age, leverage, and industry appear to have significant negative relationship. Further, board independence and firm size were found insignificant. 
Table 3: Meta-Analysis for Board Independence

\begin{tabular}{|c|c|c|c|c|c|c|c|c|c|c|c|}
\hline \multicolumn{7}{|c|}{ Correlation Coefficients for Board Independence } & \multicolumn{5}{|c|}{ Average Corrected Correlation Coefficient } \\
\hline $\begin{array}{l}\text { Sr. } \\
\text { No. }\end{array}$ & Author Name \& Year & $\begin{array}{l}\text { Sample } \\
\text { Size(N) }\end{array}$ & ROA & $\begin{array}{c}\text { Tobin's } \\
\text { Q }\end{array}$ & $\begin{array}{l}\text { Market to Book } \\
\text { Value Ratio }\end{array}$ & $\begin{array}{c}\text { Return on } \\
\text { Investment }\end{array}$ & $\begin{array}{c}\text { Sum of All } \\
\text { Ratios }\end{array}$ & $\begin{array}{l}\text { No Of } \\
\text { Ratios }\end{array}$ & $\begin{array}{l}\text { Weighted Correlation } \\
\text { Coefficient }(\mathbf{R})\end{array}$ & $\mathbf{R n}$ & $\mathbf{R c}=\mathbf{R n} / \mathbf{N}$ \\
\hline 1 & Bonn et al. (2004) & & & & & & & & & & \\
\hline & For Australian Firms & 104 & 0.254 & & -0.052 & & 0.202 & 2 & 0.101 & 10.504 & \\
\hline & For Japanese Firms & 169 & -0.028 & & -0.011 & & -0.039 & 2 & -0.0195 & -3.2955 & 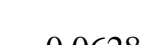 \\
\hline 2 & Andres et al. (2005) & 450 & & & -0.102 & & -0.102 & 1 & -0.102 & -45.9 & -0.0628 \\
\hline 3 & Beiner et al. (2006) & 109 & & -0.1247 & & & -0.1247 & 1 & -0.1247 & -13.5923 & \\
\hline & Total Sample Size & 832 & & & & & & & & -52.2838 & \\
\hline
\end{tabular}

Table 4: Meta-Analysis for Female Representation on Board

\begin{tabular}{|c|c|c|c|c|c|c|c|c|c|c|c|}
\hline \multicolumn{7}{|c|}{ Correlation Coefficients for Board Diversity/Board Gender } & \multicolumn{5}{|c|}{ Average Corrected Correlation Coefficient } \\
\hline $\begin{array}{l}\text { Sr. } \\
\text { No. }\end{array}$ & Author Name \& Year & $\begin{array}{l}\text { Sample } \\
\text { Size(N) }\end{array}$ & ROA & Tobin's Q & $\begin{array}{c}\text { Market to Book } \\
\text { Value Ratio }\end{array}$ & $\begin{array}{l}\text { Return on } \\
\text { Investment }\end{array}$ & $\begin{array}{c}\text { Sum of All } \\
\text { Ratios }\end{array}$ & $\begin{array}{l}\text { No Of } \\
\text { Ratios }\end{array}$ & $\begin{array}{c}\text { Weighted Correlation } \\
\text { Coefficient }(\mathbf{R})\end{array}$ & $\mathbf{R n}$ & $\mathbf{R c}=\mathbf{R n} / \mathbf{N}$ \\
\hline 1 & Erhardt et al. (2003) & & & & & & & & & & \multirow{7}{*}{0.0934} \\
\hline & For Year 1993 & 112 & 0.02 & & & 0.03 & 0.05 & 2 & 0.025 & 2.8 & \\
\hline & For Year 1998 & 112 & 0.18 & & & 0.21 & 0.39 & 2 & 0.195 & 21.84 & \\
\hline 2 & Bonn et al. (2004) & & & & & & & & & & \\
\hline & For Australian Firms & 104 & 0.028 & & 0.285 & & 0.313 & 2 & 0.1565 & 16.276 & \\
\hline & For Japanese Firms & 169 & 0.035 & & 0.03 & & 0.065 & 2 & 0.0325 & 5.4925 & \\
\hline & Total Sample Size & 497 & & & & & & & & 46.4085 & \\
\hline
\end{tabular}

\section{Table 5: Meta-Analysis for Board Size}

\begin{tabular}{|c|c|c|c|c|c|c|c|c|c|c|c|}
\hline \multicolumn{7}{|c|}{ Correlation Coefficients for Board Size } & \multicolumn{5}{|c|}{ Average Corrected Correlation Coefficient } \\
\hline $\begin{array}{l}\text { Sr. } \\
\text { No. }\end{array}$ & Correlation Table & $\begin{array}{l}\text { Sample } \\
\text { Size(N) }\end{array}$ & ROA & Tobin's Q & $\begin{array}{l}\text { Market to Book } \\
\text { Value Ratio }\end{array}$ & $\begin{array}{c}\text { Return on } \\
\text { Investment }\end{array}$ & $\begin{array}{c}\text { Sum of All } \\
\text { Ratios }\end{array}$ & $\begin{array}{l}\text { No Of } \\
\text { Ratios }\end{array}$ & $\begin{array}{l}\text { Weighted Correlation } \\
\text { Coefficient }(\mathbf{R})\end{array}$ & $\mathbf{R n}$ & $\mathbf{R c}=\mathbf{R n} / \mathbf{N}$ \\
\hline 1 & Eisenberg et al. (1998) & 879 & -0.179 & & & & -0.179 & 1 & -0.179 & -157.341 & \\
\hline 2 & Erhardt et al. (2003) & & & & & & & & & & \\
\hline & For Year 1993 & 112 & -0.03 & & & -0.04 & -0.07 & 2 & -0.035 & -3.92 & \\
\hline & For Year 1998 & 112 & 0.01 & & & 0.1 & 0.11 & 2 & 0.055 & 6.16 & \\
\hline 3 & Bonn et al. (2004) & & & & & & & & & & \\
\hline & For Australian Firms & 104 & 0.042 & & -0.182 & & -0.14 & 2 & -0.07 & -7.28 & -0.1121 \\
\hline & For Japanese Firms & 169 & -0.208 & & -0.31 & & -0.518 & 2 & -0.259 & -43.771 & \\
\hline 4 & Andres et al. (2005) & 450 & & & -0.031 & & -0.031 & 1 & -0.031 & -13.95 & \\
\hline 5 & Beiner et al. (2006) & 109 & & 0.0296 & & & 0.0296 & 1 & 0.0296 & 3.2264 & \\
\hline & Total Sample Size & 1935 & & & & & & & & -216.876 & \\
\hline
\end{tabular}

Table 6: Meta-Analysis for Firm Size

\begin{tabular}{|c|c|c|c|c|c|c|c|c|c|c|c|}
\hline \multicolumn{7}{|c|}{ Correlation Coefficients for Firm Size } & \multicolumn{5}{|c|}{ Average Corrected Correlation Coefficient } \\
\hline $\begin{array}{l}\text { Sr. } \\
\text { No. }\end{array}$ & Author Name \& Year & $\begin{array}{l}\text { Sample } \\
\text { Size }(\mathbf{N})\end{array}$ & ROA & Tobin's Q & $\begin{array}{l}\text { Market to Book } \\
\text { Value Ratio }\end{array}$ & $\begin{array}{l}\text { Return on } \\
\text { Investment }\end{array}$ & $\begin{array}{l}\text { Sum of All } \\
\text { Ratios }\end{array}$ & $\begin{array}{l}\text { No Of } \\
\text { Ratios }\end{array}$ & $\begin{array}{l}\text { Weighted Correlation } \\
\text { Coefficient }(\mathbf{R})\end{array}$ & $\mathbf{R n}$ & $\mathbf{R c}=\mathbf{R n} / \mathbf{N}$ \\
\hline 1 & Eisenberg et al. (1998) & 879 & -0.185 & & & & -0.185 & 1 & -0.185 & -162.615 & \\
\hline 2 & Erhardt et al. (2003) & & & & & & 0 & & & & \\
\hline & For Year 1993 & 112 & -0.17 & & & -0.25 & -0.42 & 2 & -0.21 & -23.52 & 0.0114 \\
\hline & For Year 1998 & 112 & -0.15 & & & 0.03 & -0.12 & 2 & -0.06 & -6.72 & \\
\hline
\end{tabular}


\begin{tabular}{|c} 
Table 7: Meta-Analysis for Board Meetings \\
Correlation Coefficients for Board Meetings
\end{tabular}

\begin{tabular}{|c|c|c|c|c|c|c|c|c|c|c|c|}
\hline \multicolumn{7}{|c|}{ Correlation Coefficients for Board Meetings } & \multicolumn{5}{|c|}{ Average Corrected Correlation Coefficient } \\
\hline Sr. No. & Author Name \& Year & $\begin{array}{l}\text { Sample } \\
\text { Size }(N)\end{array}$ & ROA & Tobin's Q & $\begin{array}{l}\text { Market to Book } \\
\text { Value Ratio }\end{array}$ & $\begin{array}{l}\text { Return on } \\
\text { Investment }\end{array}$ & $\begin{array}{c}\text { Sum of All } \\
\text { Ratios }\end{array}$ & $\begin{array}{l}\text { No Of } \\
\text { Ratios }\end{array}$ & $\begin{array}{l}\text { Weighted Correlation } \\
\text { Coefficient }(\mathbf{R})\end{array}$ & $\mathbf{R n}$ & $\mathbf{R c}=\mathbf{R n} / \mathbf{N}$ \\
\hline 1 & Andres et al. (2005) & 450 & & & 0.1 & & 0.1 & 1 & 0.1 & 45 & 01000 \\
\hline & Total Sample Size & 450 & & & & & & & & 45 & 0.1000 \\
\hline
\end{tabular}

Table 8: Meta-Analysis for Firm Age

\begin{tabular}{|c|c|c|c|c|c|c|c|c|c|c|c|}
\hline \multicolumn{7}{|c|}{ Correlation Coefficients for Firm Age } & \multicolumn{5}{|c|}{ Average Corrected Correlation Coefficient } \\
\hline Sr. No. & Author Name \& Year & $\begin{array}{l}\text { Sample } \\
\text { Size(N) }\end{array}$ & ROA & $\begin{array}{l}\text { Tobin's } \\
\text { Q }\end{array}$ & $\begin{array}{l}\text { Market to Book } \\
\text { Value Ratio }\end{array}$ & $\begin{array}{l}\text { Return on } \\
\text { Investment }\end{array}$ & $\begin{array}{c}\text { Sum of All } \\
\text { Ratios }\end{array}$ & $\begin{array}{l}\text { No Of } \\
\text { Ratios }\end{array}$ & $\begin{array}{l}\text { Weighted Correlation } \\
\text { Coefficient }(\mathbf{R})\end{array}$ & $\mathbf{R n}$ & $\mathbf{R c}=\mathbf{R n} / \mathbf{N}$ \\
\hline 1 & Eisenberg et al. (1998) & 879 & -0.13 & & & & -0.13 & 1 & -0.13 & -114.27 & \\
\hline 2 & Bonn et al. (2004) & & & & & & 0 & & & & \\
\hline & For Australian Firms & 104 & 0.131 & & -0.186 & & -0.055 & 2 & -0.0275 & -2.86 & -0.1357 \\
\hline & For Japanese Firms & 169 & -0.247 & & -0.217 & & -0.464 & 2 & -0.232 & -39.208 & \\
\hline & Total Sample Size & 1152 & & & & & & & & -156.338 & \\
\hline
\end{tabular}

Table 9: Meta-Analysis for Leverage

\begin{tabular}{|c|c|c|c|c|c|c|c|c|c|c|c|}
\hline \multicolumn{7}{|c|}{ Correlation Coefficients for Leverage } & \multicolumn{5}{|c|}{ Average Corrected Correlation Coefficient } \\
\hline $\begin{array}{l}\text { Sr. } \\
\text { No. }\end{array}$ & Author Name \& Year & $\begin{array}{l}\text { Sample } \\
\text { Size(N) }\end{array}$ & ROA & Tobin's Q & $\begin{array}{l}\text { Market to Book } \\
\text { Value Ratio }\end{array}$ & $\begin{array}{l}\text { Return on } \\
\text { Investment }\end{array}$ & $\begin{array}{l}\text { Sum of All } \\
\text { Ratios }\end{array}$ & $\begin{array}{l}\text { No Of } \\
\text { Ratios }\end{array}$ & $\begin{array}{l}\text { Weighted Correlation } \\
\text { Coefficient }(\mathbf{R})\end{array}$ & $\mathbf{R n}$ & $\mathbf{R c}=\mathbf{R} \mathbf{n} / \mathbf{N}$ \\
\hline 1 & Beiner et al. (2006) & 109 & & -0.2457 & & & -0.2457 & 1 & -0.2457 & -26.7813 & \\
\hline & Total Sample Size & 109 & & & & & & & & -26.7813 & -0.2457 \\
\hline
\end{tabular}

Table 10: Meta-Analysis for Industry

\begin{tabular}{|c|c|c|c|c|c|c|c|c|c|c|c|}
\hline \multicolumn{7}{|c|}{ Correlation Coefficients for Industry } & \multicolumn{5}{|c|}{ Average Corrected Correlation Coefficient } \\
\hline $\begin{array}{l}\text { Sr. } \\
\text { No. }\end{array}$ & Author Name \& Year & $\begin{array}{l}\text { Sample } \\
\text { Size(N) }\end{array}$ & ROA & Tobin's Q & $\begin{array}{c}\text { Market to Book } \\
\text { Value Ratio }\end{array}$ & $\begin{array}{l}\text { Return on } \\
\text { Investment }\end{array}$ & $\begin{array}{c}\text { Sum of All } \\
\text { Ratios }\end{array}$ & $\begin{array}{l}\text { No Of } \\
\text { Ratios }\end{array}$ & $\begin{array}{c}\text { Weighted Correlation } \\
\text { Coefficient }(\mathbf{R})\end{array}$ & $\mathbf{R n}$ & $\mathbf{R c}=\mathbf{R n} / \mathbf{N}$ \\
\hline 1 & Erhardt et al. (2003) & & & & & & & & & & \\
\hline & For Year 1993 & 112 & -0.22 & & & 0.04 & -0.18 & 2 & -0.09 & -10.08 & \\
\hline & For Year 1998 & 112 & -0.38 & & & -0.35 & -0.73 & 2 & -0.365 & -40.88 & -0.2275 \\
\hline & Total Sample Size & 224 & & & & & & & & -50.96 & \\
\hline
\end{tabular}




\subsection{Meta-Analysis for the Studies in Developing Countries}

In developing countries, the variables for which the data was available and were included into the analysis, are board size, CEO duality, board independence, board meetings, female representation on board, managerial ownership, ownership concentration, firm size, firm age, leverage and industry. Table 10 to Table 19 presents one by one metaanalysis of those variables. Meta-analysis results revealed that ownership concentration and firm size have significant positive relationship with firm financial performance while board meetings, managerial ownership, firm age and leverage have significant negative relationship. Further, board size, CEO duality, board independence, female representation on board and industry were found insignificant. 
Table 11: Meta-Analysis for Board Independence.

\begin{tabular}{|c|c|c|c|c|c|c|c|c|c|c|c|c|c|c|}
\hline \multicolumn{10}{|c|}{ Correlation Coefficients for Board Independence } & \multicolumn{5}{|c|}{ Average Corrected Correlation Coefficient } \\
\hline $\begin{array}{l}\text { Sr. } \\
\text { No. }\end{array}$ & Author Name \& Year & $\begin{array}{l}\text { Sample } \\
\text { Size(N) }\end{array}$ & ROA & ROE & $\begin{array}{l}\text { Tobin's } \\
\text { Q }\end{array}$ & $\begin{array}{l}\text { Market to Book } \\
\text { Value Ratio }\end{array}$ & $\begin{array}{l}\text { Earning } \\
\text { s Per } \\
\text { Share }\end{array}$ & (EVA) & $\begin{array}{l}\text { Export Intensity } \\
\text { (Ratio of Overseas } \\
\text { Sales to total Sales) }\end{array}$ & $\begin{array}{l}\text { Sum of } \\
\text { All } \\
\text { Ratios }\end{array}$ & $\begin{array}{l}\text { No Of } \\
\text { Ratios }\end{array}$ & $\begin{array}{l}\text { Weighted } \\
\text { Correlation } \\
\text { Coefficient }(\mathbf{R})\end{array}$ & Rn & $R c=R n / N$ \\
\hline 1 & Jackling \& Johl (2009) & 180 & 0.05 & & 0.04 & & & & & 0.09 & 2 & 0.045 & 8.1 & \\
\hline 2 & Sheikh et al.(2013) & 154 & -0.06 & -0.02 & & -0.1 & -0.08 & & & -0.26 & 4 & -0.065 & -10.01 & \\
\hline 3 & Fernandez et al. (2014) & 121 & 0.08 & 0.11 & -0.02 & & & & & 0.17 & 3 & 0.0567 & 6.8567 & \\
\hline 4 & Gaur et al., (2015) & 145 & 0.069 & & & & & & & 0.069 & 1 & 0.069 & 10.005 & \\
\hline 5 & Pamburai et al.(2015) & 158 & -0.0211 & & 0.0395 & & & 0.0607 & & 0.0791 & 3 & 0.0264 & 4.1659 & \\
\hline 6 & Nas \& Kalaycioglu (2016) & 221 & & & & & & & -0.01 & -0.01 & 1 & -0.01 & -2.21 & -0.0261 \\
\hline 7 & Detthamrong et al.(2017) & 3854 & -0.05 & -0.01 & & & & & & -0.06 & 2 & -0.03 & -115.62 & \\
\hline 8 & Nguyen et al.(2017) & 217 & -0.086 & & & & & & & -0.086 & 1 & -0.086 & -18.662 & \\
\hline 9 & Palaniappan G. (2017) & 275 & -0.11 & -0.101 & -0.025 & & & & & -0.236 & 3 & -0.0787 & -21.633 & \\
\hline & Total Sample Size & 5325 & & & & & & & & & & & -139.01 & \\
\hline
\end{tabular}

Table 12: Meta-Analysis for CEO Duality

\begin{tabular}{|c|c|c|c|c|c|c|c|c|c|c|c|c|c|c|}
\hline \multicolumn{10}{|c|}{ Correlation Coefficients for CEO Duality } & \multicolumn{5}{|c|}{ Average Corrected Correlation Coefficient } \\
\hline $\begin{array}{l}\text { Sr. } \\
\text { No. }\end{array}$ & Author Name \& Year & $\begin{array}{l}\text { Sample } \\
\text { Size(N) }\end{array}$ & ROA & ROE & $\begin{array}{c}\text { Tobin's } \\
\text { Q }\end{array}$ & $\begin{array}{c}\text { Market to Book } \\
\text { Value Ratio }\end{array}$ & $\begin{array}{c}\text { Earnings } \\
\text { Per } \\
\text { Share }\end{array}$ & $\begin{array}{l}\text { Profit } \\
\text { Margin }\end{array}$ & $\begin{array}{c}\text { Export Intensity } \\
\text { (Ratio of Overseas } \\
\text { Sales to total Sales) }\end{array}$ & $\begin{array}{c}\text { Sum of } \\
\text { All } \\
\text { Ratios }\end{array}$ & $\begin{array}{l}\text { No Of } \\
\text { Ratios }\end{array}$ & $\begin{array}{c}\text { Weighted } \\
\text { Correlation } \\
\text { Coefficient(R) }\end{array}$ & $\mathbf{R n}$ & $\mathbf{R c}=\operatorname{Rn} / \mathbf{N}$ \\
\hline \multirow[t]{4}{*}{1} & Abdullah (2004) & & & & & & & & & & & & & \multirow[t]{16}{*}{0.0052} \\
\hline & Panel A-1994 & 369 & 0.023 & -0.021 & & & -0.017 & 0.066 & & 0.051 & 4 & 0.0128 & 4.70475 & \\
\hline & Panel A-1995 & 369 & 0.067 & 0.024 & & & 0.003 & 0.124 & & 0.218 & 4 & 0.0545 & 20.1105 & \\
\hline & Panel A-1996 & 369 & -0.04 & -0.025 & & & -0.031 & 0.039 & & -0.057 & 4 & -0.0143 & -5.25825 & \\
\hline 2 & Haniffa \& Hudaib (2006) & 347 & 0.04 & & -0.069 & & & & & -0.029 & 2 & -0.0145 & -5.0315 & \\
\hline 3 & Jackling \& Johl (2009) & 180 & -0.02 & & -0.01 & & & & & -0.03 & 2 & -0.015 & -2.7 & \\
\hline 4 & Ujunwa (2012) & 122 & 0.009 & & & & & & & 0.009 & 1 & 0.009 & 1.098 & \\
\hline 5 & Hassan \& Halbouni (2013) & 95 & -0.202 & -0.204 & 0.077 & & & & & -0.329 & 3 & -0.1097 & -10.4183 & \\
\hline 6 & Sheikh et al.(2013) & 154 & -0.1 & -0.04 & & -0.09 & 0.005 & & & -0.225 & 4 & -0.0563 & -8.6625 & \\
\hline 7 & Fernandez et al. (2014) & 121 & & -0.04 & -0.01 & & & & & -0.05 & 2 & -0.025 & -3.025 & \\
\hline 8 & Gaur et al., (2015) & 145 & -0.032 & & & & & & & -0.032 & 1 & -0.032 & -4.64 & \\
\hline 9 & Nas \& Kalaycioglu (2016) & 221 & & & & & & & 0.03 & 0.03 & 1 & 0.03 & 6.63 & \\
\hline 10 & Detthamrong et al.(2017) & 3854 & -0.05 & 0.06 & & & & & & 0.01 & 2 & 0.005 & 19.27 & \\
\hline 11 & Nguyen et al.(2017) & 217 & -0.031 & & & & & & & -0.031 & 1 & -0.031 & -6.727 & \\
\hline \multirow[t]{2}{*}{12} & Palaniappan G. (2017) & 275 & 0.061 & 0.086 & 0.183 & & & & & 0.33 & 3 & 0.11 & 30.25 & \\
\hline & Total Sample Size & 6838 & & & & & & & & & & & 35.60067 & \\
\hline
\end{tabular}


Table 13: Meta-Analysis for Board Size

Correlation Coefficients for Board Size

Cr. Correlation Table

\begin{tabular}{|l|l|l|}
\hline Sr. & Correlation Table & Sample \\
\hline
\end{tabular}

\begin{tabular}{|l|l|} 
No. & \\
\hline 1 & Haniffa \& Hudaib (2006) \\
\hline & Jackaing \& John (2009) \\
\hline
\end{tabular}

2 Jackling \& Johl (2009)

3 Mollah et al. 2012

4 Ujunwa (2012)

5 Hassan \& Halbouni (2013)

6 Sheikh et al.(2013)

7 Fernandez et al. (2014)

8 Afrifa \& Tauringan

$8 \quad(2015)$

$9 \quad$ Gaur et al., (2015)

10 Pamburai et al.(2015)

$11 \quad$ Nas \& Kalaycioglu (2016)

12 Detthamrong et al.(2017)

13 Nguyen et al.(2017)

14 Palaniappan G. (2017)

Total Sample Size

\begin{tabular}{|l|l|l|l|l|l|l|l|l}
$\begin{array}{l}\text { Sample } \\
\text { Size(N) }\end{array}$ & ROA & ROE & $\begin{array}{l}\text { Tobin' } \\
\text { s Q }\end{array}$ & $\begin{array}{l}\text { Market to } \\
\text { Book Value } \\
\text { Ratio }\end{array}$ & $\begin{array}{l}\text { Earning } \\
\text { s Per } \\
\text { Share }\end{array}$ & $\begin{array}{l}\text { Economi } \\
\text { c Return } \\
\text { (EVA) }\end{array}$ & $\begin{array}{l}\text { Export Intensity } \\
\text { (Ratio of Overseas } \\
\text { Sales to total Sales) }\end{array}$ & $\begin{array}{l}\text { Market } \\
\text { Capitalizati } \\
\text { on }\end{array}$ \\
\hline $\mathbf{3 4 7}$ & 0.171 & & -0.094 & & & & & \\
\hline $\mathbf{1 8 0}$ & 0.09 & & 0.15 & & & & & \\
\hline $\mathbf{1 9}$ & -0.006 & 0.001 & -0.12 & & & & & \\
$\mathbf{1 2 2}$ & 0.0035 & & & & & & & \\
$\mathbf{9 5}$ & -0.139 & -0.12 & -0.099 & & & & & \\
\hline $\mathbf{1 5 4}$ & 0.1 & 0.09 & & 0.1 & 0.2 & & & \\
\hline $\mathbf{1 2 1}$ & 0.19 & 0.2 & -0.06 & & & & & \\
\hline $\mathbf{2 3 4}$ & & & -0.2113 & & & & & \\
\hline $\mathbf{1 4 5}$ & 0.146 & & & & & & & \\
\hline $\mathbf{1 5 8}$ & 0.1322 & 0.0991 & & & & -0.0042 & & \\
$\mathbf{2 2 1}$ & & & & & & & -0.06 & \\
$\mathbf{3 8 5 4}$ & 0.08 & 0.06 & & & & & & \\
\hline $\mathbf{2 1 7}$ & 0.052 & & & & & & & \\
$\mathbf{2 7 5}$ & -0.733 & -0.764 & -0.752 & & & & & \\
\hline 6142 & & & & & & & & \\
\hline
\end{tabular}

Average Corrected Correlation Coefficient

\begin{tabular}{|l|l|l|} 
Sum of All & No Of & Weighted
\end{tabular}

Ratios Ratios Correlation

6142

Table 14: Meta-Analysis for Board Meetings

\begin{tabular}{|c|c|c|c|c|c|c|c|c|c|c|c|}
\hline \multicolumn{7}{|c|}{ Correlation Coefficients for Board Meetings } & \multicolumn{5}{|c|}{ Average Corrected Correlation Coefficient } \\
\hline $\begin{array}{l}\text { Sr. } \\
\text { No. }\end{array}$ & Author Name \& Year & Sample Size(N) & ROA & ROE & Tobin's Q & $\begin{array}{l}\text { Economic } \\
\text { Return (EVA) }\end{array}$ & $\begin{array}{l}\text { Sum of All } \\
\text { Ratios }\end{array}$ & $\begin{array}{l}\text { No Of } \\
\text { Ratios }\end{array}$ & $\begin{array}{l}\text { Weighted Correlation } \\
\text { Coefficient(R) }\end{array}$ & $\mathbf{R n}$ & $\mathbf{R c}=\mathbf{R n} / \mathbf{N}$ \\
\hline 1 & Jackling \& Johl (2009) & 180 & & & -0.01 & & -0.01 & 1 & -0.01 & -1.8 & \\
\hline 2 & Fernandez et al. (2014) & 121 & -0.07 & -0.35 & -0.02 & & -0.44 & 3 & -0.1467 & -17.7467 & \\
\hline 3 & Pamburai et al.(2015) & 158 & -0.1735 & & -0.0806 & 0.1314 & -0.123 & 3 & -0.0409 & -6.4622 & -0.2452 \\
\hline 4 & Palaniappan G. (2017) & 275 & -0.491 & -0.551 & -0.638 & & -1.68 & 3 & -0.56 & -154 & \\
\hline & Total Sample Size & 734 & & & & & & & & -180.009 & \\
\hline
\end{tabular}

Table 15: Meta-Analysis for Female Representation on Board

\begin{tabular}{|c|c|c|c|c|c|c|c|c|c|c|}
\hline \multicolumn{6}{|c|}{ Correlation Coefficients for Board Diversity/Board Gender } & \multicolumn{5}{|c|}{ Average Corrected Correlation Coefficient } \\
\hline Sr. No. & Author Name \& Year & $\begin{array}{l}\text { Sample } \\
\text { Size }(\mathbf{N})\end{array}$ & ROA & ROE & Tobin's Q & $\begin{array}{l}\text { Sum of All } \\
\text { Ratios }\end{array}$ & No Of Ratios & $\begin{array}{c}\text { Weighted Correlation } \\
\text { Coefficient }(\mathbf{R})\end{array}$ & $\mathbf{R n}$ & $\mathbf{R c}=\mathbf{R n} / \mathbf{N}$ \\
\hline \multirow[t]{2}{*}{1} & Ujunwa (2012) & 122 & 0.015 & & & 0.015 & 1 & 0.015 & 1.83 & \multirow{2}{*}{0.0150} \\
\hline & Total Sample Size & 122 & & & & & & & 1.83 & \\
\hline
\end{tabular}


Table 16: Meta-Analysis for Managerial Ownership.

\begin{tabular}{|c|c|c|c|c|c|c|c|c|c|c|c|c|c|}
\hline \multicolumn{9}{|c|}{ Correlation Coefficients for Managerial Ownership } & \multicolumn{5}{|c|}{ Average Corrected Correlation Coefficient } \\
\hline $\begin{array}{l}\text { Sr. } \\
\text { No. }\end{array}$ & $\begin{array}{l}\text { Author Name \& } \\
\text { Year }\end{array}$ & $\begin{array}{l}\text { Sample } \\
\text { Size(N) }\end{array}$ & ROA & ROE & Tobin's Q & $\begin{array}{l}\text { Market to } \\
\text { Book Value } \\
\text { Ratio }\end{array}$ & $\begin{array}{l}\text { Earnings Per } \\
\text { Share }\end{array}$ & $\begin{array}{l}\text { Market } \\
\text { Share }\end{array}$ & $\begin{array}{l}\text { Sum of } \\
\text { All Ratios }\end{array}$ & $\begin{array}{l}\text { No Of } \\
\text { Ratios }\end{array}$ & $\begin{array}{l}\text { Weighted } \\
\text { Correlation } \\
\text { Coefficient }(\mathbf{R})\end{array}$ & $\mathbf{R n}$ & $R c=R n / N$ \\
\hline 1 & $\begin{array}{l}\text { Haniffa \& Hudaib } \\
\text { (2006) }\end{array}$ & 347 & 0.023 & & -0.013 & & & & 0.01 & 2 & 0.005 & 1.735 & \multirow{6}{*}{-0.0997} \\
\hline 2 & Sheikh et al.(2013) & 154 & -0.2 & -0.1 & & -0.1 & -0.2 & & -0.6 & 4 & -0.15 & -23.1 & \\
\hline 3 & Gaur et al., (2015) & 145 & -0.056 & & & & & & -0.056 & 1 & -0.056 & -8.12 & \\
\hline 4 & Khouri (2015) & 89 & & & 0.042 & & & & 0.042 & 1 & 0.042 & 3.738 & \\
\hline \multirow[t]{2}{*}{5} & Alabdullah (2016) & 109 & & & & & & -0.536 & -0.536 & 1 & -0.536 & -58.424 & \\
\hline & Total Sample Size & 844 & & & & & & & & & & -84.171 & \\
\hline
\end{tabular}

Table 17: Meta- Analysis of Firm Size

\begin{tabular}{|c|c|c|c|c|c|c|c|c|c|c|c|c|c|c|c|}
\hline \multicolumn{11}{|c|}{ Correlation Coefficient for Firm Size } & \multicolumn{5}{|c|}{ Average Corrected Correlation Coefficient } \\
\hline Author Name \& Year & $\begin{array}{l}\text { Sample } \\
\text { Size(N) }\end{array}$ & ROA & ROE & $\begin{array}{l}\text { Tobin's } \\
\text { Q }\end{array}$ & $\begin{array}{l}\text { Market } \\
\text { to Book } \\
\text { Value } \\
\text { Ratio }\end{array}$ & $\begin{array}{l}\text { Earnings } \\
\text { Per Share }\end{array}$ & $\begin{array}{l}\text { Market } \\
\text { Share }\end{array}$ & (EVA) & $\begin{array}{l}\text { Export } \\
\text { Intensity } \\
\text { (Ratio of } \\
\text { Overseas } \\
\text { Sales to total } \\
\text { Sales) } \\
\end{array}$ & $\begin{array}{l}\text { Market } \\
\text { capitalizati } \\
\text { on }\end{array}$ & $\begin{array}{l}\text { Sum of } \\
\text { All } \\
\text { Ratios }\end{array}$ & $\begin{array}{l}\text { No Of } \\
\text { Ratios }\end{array}$ & $\begin{array}{l}\text { Weighted } \\
\text { Correlation } \\
\text { Coefficient(R) }\end{array}$ & Rn & $\begin{array}{l}\mathbf{R c}= \\
\mathbf{R n} / \\
\mathbf{N}\end{array}$ \\
\hline Haniffa \& Hudaib (2006) & 347 & 0.155 & & -0.164 & & & & & & & -0.009 & 2 & -0.0045 & -1.5615 & \\
\hline Jackling \& Johl (2009) & 180 & -0.07 & & -0.36 & & & & & & & -0.43 & 2 & -0.215 & -38.7 & \\
\hline Adebiyi \& Sunday (2011) & 30 & & 0.326 & & & & & & & & 0.326 & 1 & 0.326 & 9.78 & \\
\hline Mollah et al. (2012) & 19 & -0.133 & -0.074 & -0.032 & & & & & & -0.019 & -0.258 & 4 & -0.0645 & -1.2255 & \\
\hline Ujunwa (2012) & 122 & 0.041 & & & & & & & & & 0.041 & 1 & 0.041 & 5.002 & \\
\hline Hassan \& Halbouni (2013) & 95 & 0.245 & 0.314 & -0.169 & & & & & & & 0.39 & 3 & 0.13 & 12.35 & \\
\hline Sheikh et al.(2013) & 154 & 0.3 & 0.2 & & 0.2 & 0.4 & & & & & 1.1 & 4 & 0.275 & 42.35 & \\
\hline Fernandez et al. (2014) & 121 & 0.24 & 0.07 & -0.06 & & & & & & & 0.25 & 3 & 0.0833 & 10.0833 & 0.11 \\
\hline Afrifa \& Tauringana (2015) & 234 & & & 0.0269 & & & & & & & 0.0269 & 1 & 0.0269 & 6.2946 & 97 \\
\hline Khouri (2015) & 89 & & & -0.057 & & & & & & & -0.057 & 1 & -0.057 & -5.073 & \\
\hline Pamburai et al.(2015) & 158 & 0.177 & & 0.0708 & & & & 0.2143 & & & 0.4621 & 3 & 0.1540 & 24.3373 & \\
\hline Alabdullah (2016) & 109 & & & & & & -0.074 & & & & -0.074 & 1 & -0.074 & -8.066 & \\
\hline Nas \& Kalaycioglu (2016) & 221 & & & & & & & & 0.17 & & 0.17 & 1 & 0.17 & 37.57 & \\
\hline Detthamrong et al.(2017) & 3854 & 0.1 & 0.21 & & & & & & & & 0.31 & 2 & 0.155 & 597.37 & \\
\hline Nguyen et al.(2017) & 217 & -0.059 & & & & & & & & & -0.059 & 1 & -0.059 & -12.803 & \\
\hline Palaniappan G. (2017) & 275 & 0.073 & 0.094 & 0.568 & & & & & & & 0.735 & 3 & 0.245 & 67.375 & \\
\hline
\end{tabular}


Table 18: Meta-Analysis for Ownership Concentration.

\begin{tabular}{|c|c|c|c|c|c|c|c|c|c|c|c|}
\hline \multicolumn{7}{|c|}{ Correlation Coefficients for Ownership Concentration } & \multicolumn{5}{|c|}{ Average Corrected Correlation Coefficient } \\
\hline $\begin{array}{l}\text { Sr. } \\
\text { No. }\end{array}$ & Author Name \& Year & $\begin{array}{l}\text { Sample } \\
\text { Size(N) }\end{array}$ & ROA & ROE & $\begin{array}{c}\text { Market to } \\
\text { Book Value } \\
\text { Ratio }\end{array}$ & $\begin{array}{l}\text { Earnings } \\
\text { Per Share }\end{array}$ & $\begin{array}{c}\text { Sum of } \\
\text { All } \\
\text { Ratios }\end{array}$ & $\begin{array}{l}\text { No Of } \\
\text { Ratios }\end{array}$ & $\begin{array}{c}\text { Weighted } \\
\text { Correlation } \\
\text { Coefficient(R) }\end{array}$ & Rn & $R c=R n / N$ \\
\hline 1 & Sheikh et al.(2013) & 154 & 0.1 & 0.1 & 0.1 & 0.1 & 0.4 & 4 & 0.1 & 15.4 & \multirow{4}{*}{0.0755} \\
\hline 2 & Gaur et al., (2015) & 145 & 0.062 & & & & 0.062 & 1 & 0.062 & 8.99 & \\
\hline 3 & Detthamrong et al.(2017) & 3854 & 0.09 & 0.06 & & & 0.15 & 2 & 0.075 & 289.05 & \\
\hline & Total Sample Size & 4153 & & & & & & & & 313.44 & \\
\hline
\end{tabular}

Table 19: Meta-Analysis for Firm Age

\begin{tabular}{|c|c|c|c|c|c|c|c|c|c|c|c|}
\hline \multicolumn{7}{|c|}{ Correlation Coefficients for Firm Age } & \multicolumn{5}{|c|}{$\begin{array}{l}\text { Average Corrected Correlation Coefficient } \\
\end{array}$} \\
\hline Sr. No. & Author Name \& Year & \begin{tabular}{|l|} 
Sample \\
Size(N)
\end{tabular} & ROA & ROE & Tobin's Q & $\begin{array}{l}\text { Export Intensity (Ratio } \\
\text { of Overseas Sales to } \\
\text { total Sales) }\end{array}$ & $\begin{array}{l}\text { Sum of } \\
\text { All } \\
\text { Ratios }\end{array}$ & No Of Ratios & $\begin{array}{l}\text { Weighted } \\
\text { Correlation } \\
\text { Coefficient(R) }\end{array}$ & Rn & $\mathbf{R c}=\mathbf{R n} / \mathbf{N}$ \\
\hline 1 & Jackling \& Johl (2009) & 180 & 0.06 & & -0.02 & & 0.04 & 2 & 0.02 & 3.6 & \multirow{13}{*}{-0.0392} \\
\hline 2 & Adebiyi \& Sunday (2011) & 30 & & 0.224 & & & 0.224 & 1 & 0.224 & 6.72 & \\
\hline 3 & Ujunwa (2012) & 122 & 0.223 & & & & 0.223 & 1 & 0.223 & 27.206 & \\
\hline 4 & Hassan \& Halbouni (2013) & 95 & -0.142 & -0.124 & -0.117 & & -0.383 & 3 & -0.1277 & -12.1283 & \\
\hline 5 & Fernandez et al. (2014) & 121 & 0.13 & 0.03 & 0.11 & & 0.27 & 3 & 0.09 & 10.89 & \\
\hline 6 & Afrifa \& Tauringana (2015) & 234 & & & 0.0821 & & 0.0821 & 1 & 0.0821 & 19.2114 & \\
\hline 7 & Gaur et al., (2015) & 145 & 0.135 & & & & 0.135 & 1 & 0.135 & 19.575 & \\
\hline 8 & Khouri (2015) & 89 & & & 0.001 & & 0.001 & 1 & 0.001 & 0.089 & \\
\hline 9 & Nas \& Kalaycioglu (2016) & 221 & & & & -0.12 & -0.12 & 1 & -0.12 & -26.52 & \\
\hline 10 & Detthamrong et al.(2017) & 3854 & -0.07 & -0.08 & & & -0.15 & 2 & -0.075 & -289.05 & \\
\hline 11 & Nguyen et al.(2017) & 217 & -0.093 & & & & -0.093 & 1 & -0.093 & -20.181 & \\
\hline \multirow[t]{2}{*}{12} & Palaniappan G. (2017) & 275 & 0.481 & -0.035 & 0.01 & & 0.456 & 3 & 0.152 & 41.8 & \\
\hline & Total Sample Size & 5583 & & & & & & & & -218.788 & \\
\hline
\end{tabular}

\section{Table 20: Meta-Analysis for Industry.}

\begin{tabular}{|c|c|c|c|c|c|c|c|c|c|c|c|c|}
\hline \multicolumn{8}{|c|}{ Correlation Coefficients for Industry } & \multicolumn{5}{|c|}{ Average Corrected Correlation Coefficient } \\
\hline $\begin{array}{l}\text { Sr. } \\
\text { No. }\end{array}$ & Author Name \& Year & $\begin{array}{l}\text { Sample } \\
\text { Size(N) }\end{array}$ & ROA & ROE & Tobin's Q & $\begin{array}{l}\text { Market } \\
\text { Share }\end{array}$ & $\begin{array}{l}\text { Export Intensity } \\
\text { (Ratio of Overseas } \\
\text { Sales to total Sales) }\end{array}$ & $\begin{array}{l}\text { Sum of } \\
\text { All } \\
\text { Ratios }\end{array}$ & $\begin{array}{l}\text { No Of } \\
\text { Ratios }\end{array}$ & $\begin{array}{l}\text { Weighted } \\
\text { Correlation } \\
\text { Coefficient }(\mathbf{R})\end{array}$ & $\mathbf{R n}$ & $\mathbf{R c}=\mathbf{R n} / \mathbf{N}$ \\
\hline 1 & Hassan \& Halbouni (2013) & 95 & -0.144 & -0.09 & 0.088 & & & -0.146 & 3 & -0.0487 & -4.6233 & \\
\hline 2 & Alabdullah (2016) & 109 & & & & 0.081 & & 0.081 & 1 & 0.081 & 8.829 & \\
\hline 3 & Nas \& Kalaycioglu (2016) & 221 & & & & & 0.06 & 0.06 & 1 & 0.06 & 13.26 & 0.0491 \\
\hline 4 & Nguyen et al.(2017) & 217 & 0.065 & & & & & 0.065 & 1 & 0.065 & 14.105 & \\
\hline & Total Sample Size & 642 & & & & & & & & & 31.57067 & \\
\hline
\end{tabular}


Table 21: Meta-Analysis for Leverage.

\begin{tabular}{|c|c|c|c|c|c|c|c|c|c|c|c|c|c|}
\hline 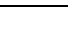 & 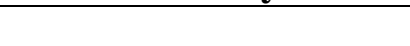 & Co & ation $\mathrm{Co}$ & ficients f & Leverage & & & & & Average $\mathrm{C}$ & orrected Correlation & Coefficient & \\
\hline $\begin{array}{l}\text { Sr. } \\
\text { No. }\end{array}$ & Author Name \& Year & $\begin{array}{l}\text { Sample } \\
\text { Size(N) }\end{array}$ & ROA & ROE & $\begin{array}{l}\text { Tobin's } \\
\text { Q }\end{array}$ & $\begin{array}{l}\text { Market to } \\
\text { Book Value } \\
\text { Ratio }\end{array}$ & $\begin{array}{l}\text { Earnings } \\
\text { Per Share }\end{array}$ & $\begin{array}{l}\text { Economic } \\
\text { Return } \\
\text { (EVA) }\end{array}$ & $\begin{array}{l}\text { Sum of } \\
\text { All } \\
\text { Ratios }\end{array}$ & $\begin{array}{l}\text { No Of } \\
\text { Ratios }\end{array}$ & $\begin{array}{l}\text { Weighted } \\
\text { Correlation } \\
\text { Coefficient }(\mathbf{R})\end{array}$ & $\mathbf{R n}$ & $R c=R n / N$ \\
\hline 1 & Jackling \& Johl (2009) & 180 & -0.46 & & -0.21 & & & & -0.67 & 2 & -0.335 & -60.3 & \\
\hline 2 & Hassan \& Halbouni (2013) & 95 & 0.037 & 0.033 & 0.036 & & & & 0.106 & 3 & 0.0353 & 3.3567 & \\
\hline 3 & Sheikh et al.(2013) & 154 & -0.4 & -0.2 & & 0.01 & -0.2 & & -0.79 & 4 & -0.1975 & -30.415 & \\
\hline 4 & Afrifa \& Tauringana (2015) & 234 & & & -0.0004 & & & & -0.0004 & 1 & -0.0004 & -0.0936 & \\
\hline 5 & Khouri (2015) & 89 & & & 0.012 & & & & 0.012 & 1 & 0.012 & 1.068 & -0.1272 \\
\hline 6 & Pamburai et al.(2015) & 158 & -0.138 & & -0.1212 & & & 0.0611 & -0.1981 & 3 & -0.0660 & -10.4333 & \\
\hline 7 & Nguyen et al.(2017) & 217 & -0.305 & & & & & & -0.305 & 1 & -0.305 & -66.185 & \\
\hline 8 & Palaniappan G. (2017) & 275 & -0.025 & -0.063 & -0.08 & & & & -0.168 & 3 & -0.056 & -15.4 & \\
\hline & Total Sample Size & 1402 & & & & & & & & & & -178.402 & \\
\hline
\end{tabular}




\section{Results and Discussion}

Table 22 summarizes the results on the impact of CG on firm financial performance in both developed and developing countries separately. Board independence is found to have significant negative relationship with firm financial performance in both developed $(\mathrm{Rc}=-0.0628, \mathrm{P}<0.10)$ and developing countries $(\mathrm{Rc}=-0.026, \mathrm{P}<0.10)$. The significant result may be due to the reason that there is nominal representation of independent directors on the board. It shows that independent directors on boards appear to negatively contribute to upgrade firm performance. This result is contradictory to the prediction of agency theory which argue that effective boards largely consists of independent and outside directors (Dalton et al. 1999). The lack of independent directors on boards makes the firm difficult to respond to critical issues in the top management. Further, independent directors increase the monitoring ability of top level executives, largely found involved in corporate scams and also control the agency problems of the company. The result revealed a significant negative relationship between board size and firm financial performance in developed countries $(\mathrm{Rc}=-0.1121, \mathrm{P}<0.05)$ and insignificant in developing countries $(\mathrm{Rc}=0.01672, \mathrm{P}>0.05)$. This result is contradictory to the predictions of resource dependence theory which suggests that large board is necessary to increase the performance of companies and to acquire resource from external environment. Contrary to the findings of developing countries where board meeting and firm performance have significant negative relationship $(\mathrm{Rc}=-0.2452$, $\mathrm{P}<0.05)$, number of board meeting appear to positively contributes towards financial performance in developed countries $(\mathrm{Rc}=0.1000, \mathrm{P}<0.05)$. Thus board meeting have differential impact in both developed and developing countries. The positive result between board meeting and firm performance supports the view that a greater constancy of board meetings is likely to result in remarkable performance (Lipton and Lorsch, 1992).In addition to this, Conger et al. (1998) outlined that "board meeting time is an important resource that helps in improving the effectiveness of board". Contrary to the results of developing countries where female representation on board has insignificant relationship with firm performance $(\mathrm{Rc}=0.0150, \mathrm{P}>0.05)$, the female representation on board cause to uplift the firm performance in developed countries $(\mathrm{Rc}=0.0934, \mathrm{P}<0.05)$. In addition to the above, ownership concentration $(\mathrm{Rc}=$ $0.076, \mathrm{P}<0.05)$ and managerial ownership $(\mathrm{Rc}=-0.100, \mathrm{P}<0.05)$ appear to play differential role towards corporate financial performance in developing countries. The role of these variables has not been examined in developed countries due to the non availability of data.

Table 22: Results for the Relationship between CG and Firm Financial Performance

\begin{tabular}{|l|c|c|c|c|c|c|}
\hline \multirow{2}{*}{ Variables/Proxies } & \multicolumn{3}{c|}{ Developed Countries } & \multicolumn{3}{c|}{ Developing Countries } \\
\cline { 3 - 8 } & $\mathbf{N}$ & $\mathbf{R c}$ & $\mathbf{P - V a l u e}$ & $\mathbf{N}$ & $\mathbf{R c}$ & P-Value \\
\hline CG Proxies & & & & & & \\
\hline Board Independence & 832 & -0.063 & 0.074 & 5325 & -0.026 & 0.058 \\
\hline CEO Duality & 0 & 0 & 0 & 6838 & 0.005 & 0.667 \\
\hline Board Size & 1935 & -0.112 & 0.000 & 6142 & 0.017 & 0.191 \\
\hline Board Meetings & 450 & 0.100 & 0.034 & 734 & -0.245 & 0.000 \\
\hline Female Representation on Board & 497 & 0.093 & 0.037 & 122 & 0.015 & 0.870 \\
\hline Managerial Ownership & 0 & 0 & 0 & 844 & -0.100 & 0.004 \\
\hline Ownership Concentration & 0 & 0 & 0 & 4153 & 0.076 & 0.000 \\
\hline Control Variable & & & & & & \\
\hline Firm Size & 1553 & 0.011 & 0.654 & 6225 & 0.120 & 0.000 \\
\hline Firm Age & 1152 & -0.136 & 0.000 & 5583 & -0.039 & 0.004 \\
\hline Financial Leverage & 109 & -0.246 & 0.010 & 1402 & -0.127 & 0.000 \\
\hline Industry & 224 & -0.228 & 0.000 & 642 & 0.049 & 0.214 \\
\hline \multicolumn{7}{|l|}{} \\
\hline
\end{tabular}

With regards to control variables, the results showed a significant positive relationship $(\mathrm{Rc}=0.1197, \mathrm{P}<0.05)$ of firm size with firm performance in developing countries while it was found insignificant in developed countries. This suggests that large firm size positively contributes to firm financial performance. Further firm age and financial leverage were found to have significant negative relationship with firm financial performance in both developed and developing countries.

\section{Conclusion}

Based on the meta-analysis of 67 empirical research papers, this research extends prior reviews / meta-analysis studies by examining the impact of CG mechanisms on firm financial performance. Further, this research contributes to the existing literature by making comparison of usefulness of CG mechanisms in developed and developing 
countries. The results showed that CG mechanisms appear to play differential role in driving corporate financial performance in both developed and developing countries. In developed countries board meeting and female representation on board has significant positive impact on financial performance while board independence and board size has significant negative relationship. Inconsistent with the results of developed countries, firm performance appear to be negatively associated with board independence, board meeting, and managerial ownership and positive associated with ownership concentration. This difference can be attributed to different social, political, and cultural factors in both developed and developing countries. The same has been echoed by Ali et al. (2017). This research is not free limitations. Firstly, this research relied on existing empirical research studies for data collection purpose and ignored other variables and characteristics of $\mathrm{CG}$ due to the non-availability of required information. Therefore, this research does not provide comprehensive view on the impact of CG on firm performance. Further, this research is limited to studies published in journals enlisted with ABS ranking 2015. Therefore future research should incorporate other authentic journals /empirical research papers in to the analysis.

\section{References}

Abdullah, S.N. (2004),"Board composition, CEO duality and performance among Malaysian listed companies", Corporate Governance: The international journal of business in society, 4(4), $47-61$.

Abor, J., (2007), "Corporate governance and financing decisions of Ghanaian listed firms", Corporate Governance: The International Journal of Business in Society, 7(1), 83-92.

Abor, J., \&Biekpe, N. (2007). Corporate governance, ownership structure and performance of SMEs in Ghana: implications for financing opportunities.Corporate Governance, 7(3), 288-300.

Abdullah, H. and Valentine, B. (2009), “Fundamental and ethics theories of corporate governance”, Middle Eastern Finance and Economics, 4(4), 88-96.

Adebiyi,A., Sunday, K., (2011). "Ownership structure and firm performance:evidence from Nigerian listed companies". Corporate Ownership \& Control, 8(4),391-400.

Afrifa, G. A., \&Tauringana, V. (2015). "Corporate governance and performance of UK listed small and medium enterprises". Corporate Governance: The international journal of business insociety, 15(5), 719-733

Agrawal, A. and Chadha, S. (2005). "Corporate Governance and Accounting Scandals", Journal of Law and Economics, 48(2), 371-406.

Alabdullah, T. T. Y. (2018) "The relationship between ownership structure and firm financial performance: Evidence from Jordan", Benchmarking: An International Journal, 25(1), 319-333.

Ali, R., Lynch, R.,Melewar, T.C., Jin, Z. (2015) "The moderating influence on the relationship of corporate reputation with its antecedent and consequences; A meta analytic review”, Journal of Business Research, 68(5), 11051117.

Ali, W., Frynas, J. G., \& Mahmood, Z. (2017). Determinants of corporate social responsibility (CSR) disclosure in developed and developing countries: A literature review. Corporate Social Responsibility and Environmental Management, 24(4), 273-294.

Alves, S. (2014). "The Effect of Board Independence on the Earnings Quality: Evidence from Portuguese Listed Companies". Australasian Accounting Business and Finance Journal, 8(3), 23-44.

Andres, P. D., Azofra, V., \& Lopez, F. (2005). "Corporate boards in OECD countries: Size, composition, functioning and effectiveness". Corporate Governance: An International Review, 13(2), 197-210.

Arora, A., Sharma, C. (2016),"Corporate governance and firm performance in developing countries: evidence from India", Corporate Governance, 16(2), $420-43$.

Baysinger, B. D. and Zardkoohi, A. (1986) Technology, Residual Claims and Corporate Control, Journal of Law, Economics and Organization, 2(2), 339-344.

Banerji, K. and Sambharya, R. B. (1996) Vertical Keiretsu and International Market Entry: The Case of the Japanese Automobile Ancillary Industry, Journal of International Business Studies, 27(1), 89-114.

Bonn, I. (2004), “Board structure and firm performance: evidence from Australia', Journal of the Australia and New Zealand Academy of Management, 10(1), 14-24.

Basyith, A., Fauzi, F. and Idris, M. (2015). "The impact of board structure and ownership structure on firm performance: an evidence from blue chip firms listed in Indonesian stock exchange", Corporate Ownership \& Control, 12(4), 344-351.

Baysinger, R. D. and Butler, H. N. (1985) "Corporate Governance and the Board of Directors: Performance Effects of Changes in Board Composition", Journal of Law, Economics and Organization, 1(1),101-124. 
Berg, S.V. and Smith, S.R. (1978), "CEO \& Board Chairman: a quantitative study of dual versus unitary board leadership", Directors and Boards, 3(1), 34-39.

Berthelot, S., Francoeur, C. and Labelle, R. (2012),"Corporate governance mechanisms, accounting results and stock valuation in Canada", International Journal of Managerial Finance, 8 (4), 332 - 343.

Beiner, S.,W. Drobetz, M.M. Schmid, and H. Zimmermann. 2006. An integrated framework of corporate governance and firm valuation. European Financial Management,12(2), 249-283.

Bhagat, S., \& Bolton, B. (2013).“Director Ownership, Governance, and Performance”. Journal of Financial \& Quantitative Analysis, 48(1),105-135.

Bokpin, G.A. and Arko, A.C., (2009). "Ownership structure, corporate governance and capital structure decisions of firms:Empirical evidence from Ghana", Studies in Economics and Finance, 26(4), 246-256.

Boyd, B.K. (1995), “CEO duality and firm performance: a contingency model”, Strategic Management Journal, 16(4), 301-12.

Buallay, A., Hamdan, A., and Zureigat, Q. (2017) "Corporate Governance and Firm Performance: Evidence from Saudi Arabia", Australasian Accounting, Business and Finance Journal, 11(1), 78-98.

Carter, D.A., D’Souza, F., Simkins, B.J. and Simpson, W.G. 2010, 'The Gender and Ethnic Diversity of US Boards and Board Committees and Firm Financial Performance', CorporateGovernance: An International Review, 18 (5), 396-414.

Carter, D.A., Simkins, B.J. and Simpson, W.G. (2003), "Corporate governance, board diversity, and firm value", Financial Review, 38(1), 33-53.

Christensen, J., Kent, P. and Stewart, J. (2010), "Corporate governance and company performance in Australia", Australian Accounting Review, 20(4), 372-86.

Carpenter, M.A. and Westphal, J.D. (2001), "The strategic context of external network ties: examining the impact of director appointments on board involvement in strategic decision making", Academy of Management, 44(4), 639-660.

Claessens, S., Djankov, S., Fan, J.P.H. and Lang, L.H.P. (2002), "Disentangling the incentive and entrenchment effects of large shareholders", The Journal of Finance, 57(6), 2741-71.

Conyon, M. and Peck, S. (1998), "Board size and corporate performance: evidence from European countries", The European Journal of Finance, 4(3), 291-304.

Conger, J., Finegold, D., Lawler III, E., 1998.Appraising Boardroom Performance. Harvard Business Review 76, 136148.

Coles, J., Daniel, N. and Naveen, L. (2008), “'Boards: Does one size fit all?’, Journal of Financial Economics, Vol. 87(2), 329-56.

Combs, J.G., Ketchen, D.J., Jr., Crook, T.R., \& Roth, P.L. (2011). “Assessing cumulative evidence within 'macro' research: Why meta-analysis should be preferred over vote counting”. Journal of Management Studies, 48(1), 178-197.

Coleman, A.K., Adjasi, C.K. and Abor, J. (2006-2007). “Corporate governance and firm performance: evidence from ghanaian listed companies", Corporate Ownership \& Control, 4(2), 123-132.

Davis, J.H., Schoorman, F.D. and Donaldson, L. (1997), "Toward a stewardship theory of management", Academy of Management Review, 22(1), 20-47.

Donaldson, L. (1990) The Ethereal Hand: Organizational Economics and Management Theory, Academy of Management Review, 15, 369-381.

Donaldson, L. and Davis, J. H. (1991) Stewardship Theory or Agency Theory: CEO Governance and Shareholder Returns, Australian Journal of Management, 16, 49-64.

Donaldson, L. and Davis, J. H. (1994) Boards and Company Performance - Research Challenges the Conventional Wisdom, Corporate Governance: An International Review, 2, 151-160.

Donaldson, T. and Preston, L. E. (1995) The Stakeholder Theory of the Corporation: Concepts, Evidence, and Implications, Academy of Management Review, 20, 65-91.

Davis, J.H., Schoorman, F.D. and Donaldson, L. (1997), "Toward a stewardship theory of management", Academy of Management. The Academy of Management Review, 22(1), 20-47.

Denis, D.J., Denis, D. and Sarin, A. (1997), “Agency problems, equity ownership, and corporate Diversification”, Journal of Finance, 52(1), 135-160.

Dalton, D.R., Daily, C.M., Ellstrand, A.E. and Johnson, J.L. (1999), "Number of directors and financial performance: 
a meta-analysis", The Academy of Management Journal, 42(6), 674-686.

Dalton, D.R., Daily, C.M., Ellstrand, A.E. and Johnson, L. (1998), "Meta-analytic reviews of board composition, leadership structure, and financial performance", Strategic Management Review, 19(3), 269-290.

Darko, J., Aribi, Z.A. and Uzonwanne, G.C. (2016),"Corporate governance: the impact of director and board structure, ownership structure and corporate control on the performance of listed companies on the Ghana stock exchange", Corporate Governance: The International Journal of Business in Society, 16(2), 259-277.

Ehikioya, B.I. (2009), "Corporate governance structure and firm performance in developing Economies: evidence from Nigeria", Corporate Governance: The International Journal of Business in Society, 9(3), 231-243.

Eisenberg, T., Sundren, S. and Wells, M. (1998), "Larger board size and decreasing firm value in small firms", Journal of Financial Economics, 48(1), 35-54.

Elsayed, K. 2007. “Does CEO duality really affect corporate performance?”,Corporate Governance: An International Review, 15,1203-1214.

Erhardt, N. L., Werbel, J., \&Shrader, C. B. (2003), "Board of director diversity and firm financial performance". Corporate Governance:An International Review, 11(2),102-111.

Essen, M.V. \&Oosterhout, J.H. \& Carney, M. (2012). "Corporate boards and the performance of Asian firms: A metaanalysis", Asia Pacific Journal of Management, 29, 873-905.

Freeman, R. E. and Evan, W. M. (1990) Corporate Governance: A Stakeholder Interpretation, the Journal of Behavioral Economics, 19, 337-359.

Frooman, J. (1999) Stakeholder Influence Strategies, Academy of Management Review, 24, 191-205.

Fama\&Jensen, M. C. (1993) "The Modern Industrial Revolution, Exit, and the Failure of Internal Control Systems", Journal of Finance, 48(3), 831-880.

Fama, E. and Jensen, M. (1983), 'Separation of ownership and control', Journal of Law and Economics, 26(2), 30125.

Fernandez, M.R., Alonso, S.F. and Rodriguez, J.R. (2014), "Board characteristics and firm performance in Spain", Corporate Governance, 14(4), 485 - 503.

Fama, E. F. \& Jensen, M. C. 1983.Separation of ownership and control. Journal of Law and Economics, 26, $301-325$.

Finkelstein, L. M., Burke, M. J., \&Raju, N. 1995. Age discrimination in simulated employment contexts: An integrative analysis. Journal of Applied Psychology, 80,652-663.

Gaur, S.S., Bathula, H. and Singh, D.(2015),"Ownership concentration, board characteristics and firm performance", Management Decision, 53(5), 911 - 931.

Gedajlovic, E., Shapiro, D.M. (2002). "Ownership Structure and Firm Profitability in Japan”,Academy of Management Journal, 2002, 45(2), 565-575.

Gompers, P.A., Ishii, J.L. and Metrick, A. (2003), “ Corporate governance and equity prices', Quarterly Journal of Economics, 118(1), 107-55.

Guest, P.M. (2009), "The impact of board size on firm performance: evidence from the UK", The European Journal of Finance, 15(4), 385-404.

Gupta, P.P., Kennedy, D.B. and Weaver, S.W. (2009), “Corporate governance scores, Tobin's Q and equity prices: evidence from Canadian capital markets”, Corporate Ownership and Control Journal, 6(3), 293-307.

Goodstein, J., Gautam, K. and Boeker,W. 1994, "The Effects of Board Size and Diversity on Strategic Change", Strategic Management Journal, 15 (3), 241-50.

Hermalin, B.E. and Weisbach, M.S. (1988), "The determinants of board composition", RAND Journal of Economics, 19(4), 589-606.

Haat, M.H., Abdul Rahman, R. and Mahenthiran, S. (2008), "Corporate governance, transparency and performance of Malaysian companies", Managerial Auditing Journal, 23(8), 744-778.

Haniffa, R., and Hudaib, M. (2006). "Corporate governance structure and performance of Malaysian listed companies". Journal of Business Finance \& Accounting,33(7) \& (8), 1034-1062.

Hassan, M.K., Halbouni, S.S. (2013)“Corporate governance, economic turbulence and financial performance of UAE listed firms", Studies in Economics and Finance, 30(2), 118-138.

Hunter, J. E., \& Schmidt, F. L.1990. Methods of meta-analysis: Correcting error and bias in research findings. Newbury Park, CA: Sage, American Journal of Clinical Hypnosis, 43(1), 81-82.

Huse, M. and Solberg, A.G. (2006), "Gender related boardroom dynamics: how Scandinavian women make and can make contributions on corporate boards', Women in Management Review, 21(2), 113-30. 
Ilina,Y., Berezinets,I., Cherkasskaya, A. (2017) "Board structure, board committees and corporate performance in Russia", Managerial Finance, 43(10), 1073-1092.

Jensen, M.C. and Meckling, W.H. (1976), "Theory of the firm: managerial behavior, agency costs and ownership structure", Journal of Financial Economics, 3(4), 305-360.

Johnson, J.L., Daily, C.M. and Ellstrand, A.E. (1996), "Boards of directors: a review and research agenda", Journal of Management, 22(3), 409-438.

Jensen, M. 1993. The modern industrial revolution, exit and the failure of internal control systems.Journal of Finance, 48, 831-880.

Jackling, B. and Johl, S. (2009), "Board structure and firm performance: evidence from India's top companies", Corporate Governance: An International Review, 17(4), 492-509.

Khouri, R.A. (2015) "Corporate governance and firms value in emerging markets: the case of jordan" In Corporate Governance: Advances in Financial Economics, 11, 31 - 50.

Koontz, H. (1967), The Board of Directors and Effective Management, McGraw-Hill, New York, NY.

Klein, A. (1998) Firm Performance and Board Committee Structure, Journal of Law and Economics, 41, $275-303$.

Kumar, N., \& Singh, J. P. (2013). Effect of board size and promoter ownership on firm value: some empirical findings from India. Corporate Governance, 13(1), 88-98.

Lipton, M. \&Lorsch, J. 1992.A modest proposal for improved corporate governance. Business Lawyer, 48, 59-77.

Laing, D. and Weir, C.M. (1999),“Governance structures, size and corporate performance in UK firms”, Management Decision, 37(5), 457-464.

Lam, T.Y. and Lee, S.K. (2008), "CEO duality and firm performance: evidence from Hong Kong”, Corporate Governance, 8(3), 299-316.

Linck, J., Netter, J. and Yang, T. (2008), “the determinants of board structure', Journal of Financial Economics, 87(2), 308-28.

Lam, T.Y., Lee, S.K. (2012),"Family ownership, board committees and firm performance: evidence from Hong Kong", Corporate Governance: The international journal of business in society, 12(3), 353 - 366.

Lei, A.C. \& Song, F.M. (2012) "Board structure, corporate governance and firm value: evidence from Hong Kong", Applied Financial Economics, 22(15), 1289-1303.

Li, K., Lu, L., Mittoo,U.R. \& Zhang, Z., (2015),'Board Independence, Ownership Concentration and Corporate Performance - Chinese Evidence", InternationalReview of Financial Analysis, 41, 162-175.

Mizruchi, M. S. (1983). 'Who controls whom? An examination of the relation between management and boards of directors in large corporations', Academy of Management Review, 8(3), 426-435.

Mizruchi, M. S. and Stearns, L. B. (1988) A Longitudinal Study of the Formation of Interlocking Directorates, Administrative Science Quarterly, 33, 194-210.

Manawaduge, A. and Zoysa, A.D.(2013). "The structure of corporate ownership and firm performance: Sri lankan evidence", Corporate Ownership \& Control, 11(1), 723-734.

McMullen, D.A. (1996), “Audit committee performance: an investigation of consequences associated with audit committees", Auditing: A Journal of Practice and Theory, 15(1), 87-103.

Meca, E.G., Ballesta,J.P.S., (2011) "Firm value and ownership structure in the Spanish capital market", Corporate Governance: The international journal of business in society, 11(1), 41-53.

Monks, R.A. and Minow, N. (2004), Corporate Governance, Blackwell, Malden, MA.

McGrath, J. (2009), "How CEOs work', How Stuff Works, available at: http://money.howstuffworks.com/ ceo.htm (accessed January 15, 2011).

Nas, T.I., Kalaycioglu, O. (2016),"The effects of the board composition, board size and CEO duality on export performance: evidence from Turkey", Management Research Review, 39(11), 1374-1409.

Nguyen, T.T., Evans, E. and Lu, M., (2017) "Independent directors, ownership concentration and firm performance in listed companies: Evidence from Vietnam", Pacific Accounting Review, 29(2), 204 -226.

Narwal, K.P., Jindal, S. (2015). "The Impact of Corporate Governance on the Profitability: An Empirical Study of Indian Textile Industry", International Journal of Research in Management, Science \& Technology, 3(2), 8185 .

Nyamongo, E.M., Temesgen, k., (2013) "The effect of governance on performance of commercial banks in Kenya: apanel study", Corporate Governance: The international journal of business in society, 13(3), 236-248. 
Pearce, J.A. II and Zahra, S.A. (1992), "Board composition from a strategic contingency perspective", Journal of Management $\quad$ Studies, $411-438$. Patricia B. Abels, Joseph T. Martelli, (2013) "CEO duality: how many hats are too many?", Corporate Governance: The international journal of business in society, 13(2), 135-147,

Palaniappan G., (2017) "Determinants of corporate financial performance relating to board characteristics of corporate governance in Indian manufacturing industry: An empirical study", European Journal of Management and Business Economics, 26(1), 67-85.

Pamburai, H.H., Chamisa, E., Abdulla, C. \& Smith, C., (2015) "An analysis of corporate governance and company performance: a South African perspective”,South African Journal of Accounting Research,29(2), 115-131.

Pfeffer, J. (1972), "Size and composition of corporate boards of directors: the organization and its environment', Administrative Science Quarterly, 17(2), 218-28.

Rashid, A. (2010), "CEO duality and firm performance: evidence from a developing country",Corporate Ownership and Control, 8(1), 163-175.

Rashid, K., Islam, S.M.N., (2013),"Corporate governance, complementarities and the value of a firm in an emerging market: the effect of market imperfections", Corporate Governance: The international journal of business in society, 13(1), $70-87$.

Rashid, A.; De Zoysa, A.; Lodh, S.; and Rudkin, K.," Board Composition and Firm Performance: Evidence from Bangladesh", Australasian Accounting, Business and Finance Journal, 4(1), 76-95.

Rechner, P.L. and Dalton, D.R. (1991), "CEO duality and organizational performance: a longitudinal analysis", Strategic Management Journal, 12(2), 155-160.

Suto, M., 2003. Capital structure and investment behaviour of Malaysian firms in the 1990s: a study of corporate governance before the crisis. Corporate Governance: AnInternational Review, 11, 25-39.

Sherman, H., Kashlak, R. and Maheshkumar, J. (1998), "The effect of the board and executivescommittee characteristics on the degree of internationalization", Journal of International Management, 4, 311-335.

Short, H. and K. Keasey (1999), 'Managerial Ownership and the Performance of Firms: Evidence from the UK', Journal of Corporate Finance, 5, 79-101.

Singh, D.A. and Gaur, A.S. (2009), "Business group affiliation, firm governance and firm

performance: evidence from China and India", Corporate Governance: An International

Review, 17(4), 411-425.

Sabbaghi, O. (2016) "Corporate governance in China: a review", Corporate Governance: The International Journal of Business in Society, 16(5), 866-882.

Shan, Y., McIver, R.P., 2011. "Corporate Governance Mechanisms and Financial Performance in China: Panel Data Evidence on Listed Non-Financial Companies", Asia Pacific Business Review, 17(3), 301-324.

Sheikh, N.A, Wang, Z. and Khan, S. (2013). "The impact of internal attributes of corporate governance on firm performance-Evidence from Pakistan". International Journal of Commerce and Management,23(1), 38-55.

Ujunwa, A. (2012). "Board characteristics and the financial performance of Nigerian quoted firms". Corporate Governance: The international journal of business in society, 12(5), 656-674.

Vafeas, N., and E.Theodorou. 1998. "The relationship between board structure and firm performance in the UK". British Accounting Review, 30, 383-407.

Vafeas, N. 1999.Board meeting frequency and firm performance. Journal of Financial Economics, 53, $113-142$.

Vafaei, A., Ahmed, K. and Mather, P. (2015),"Board Diversity and Financial Performance in the Top 500 Australian Firms". Australian Accounting Review, 25,413-427.

Walsh, J. P. and J. K. Seward (1990). 'On the efficiency of internal and external corporate control mechanisms', Academy of Management Review, 15, 421-458.

Wahba, H. (2015),"The joint effect of board characteristics on financial performance: empirical evidence from Egypt", Review of Accounting and Finance, 14(1), 20-40.

Xu, L., McIver, R.P., Shan, Y.G. and Wang, X., (2016),"Governance and Performance in China's Real Estate Sector", Managerial Finance, Vol. 42(6), 585-603.

Yammeesri , J., Herath, S.k. (2010), "Board characteristics and corporate value: evidence from Thailand", Governance: The international journal of business in society, 10(3), 279-292.

Yermack, D. (2006) "Board members and company value”, Financial Markets Portfolio Management, 20(1), 33-47.

Zulkafli, A.H., Samad, F.A. "Corporate Governance and Performance of Banking Firms: Evidence from Asian Emerging Markets" In Issues in Corporate Governance and Finance, 12, 49-74. 